\title{
Relationship between GLOBE organizational culture dimensions, job satisfaction and leader-member exchange in Serbian organizations
}

\author{
Jelena Vukonjanski, Milan Nikolić, Olga Hadžić, Edit Terek, Milena \\ Nedeljkovic ${ }^{* *}$
}

The aim of this paper is to investigate the relationships between GLOBE organizational culture dimensions, facets of job satisfaction and the leadermember exchange relation in Serbian organizations. One of the most important aspects of this study was the demonstration of the utility of applying more general cultural theory to the study of organizational cultures. The sample consists of 256 middle managers in Serbian organizations. The results obtained proved that organizational culture dimensions are significant predictors of all facets of job satisfaction and that relations between some organizational culture dimensions and facets of job satisfaction are moderated by the leader-member exchange (LMX) variable. Our results may be of interest for human resource managers in Serbian organizations as well as for foreign investors.

\footnotetext{
Manuscript received: 19.07.11, accepted: 22.04 .12 (2 revisions)

** Jelena Vukonjanski, M.Sc., Dept. of Management, University of Novi Sad, Technical Faculty "Mihajlo Pupin", Zrenjanin, Serbia. Main research areas: Organizational Culture, Job Satisfaction, Knowledge Management and Human Resource Management. Corresponding address: jelena.vukonjanski@gmail.com

Milan Nikolić, Assoc. Prof., Dept. of Management, University of Novi Sad, Technical Faculty "Mihajlo Pupin”, Zrenjanin, Serbia. Main research areas: Quantitative methods in management with particular focus on business decision making, product development, reengineering, benchmarking, organizational culture and public relations.

Olga Hadžić, Full Prof., Dept. of Mathematics, Dept. of Geography, Tourism and Hotel Management, Faculty of Science, University of Novi Sad. Main research areas: Statistics, Organizational Behavior and Organizational Psychology.

Edit Terek, M.Sc., Dept. of Management, University of Novi Sad, Technical Faculty "Mihajlo Pupin", Zrenjanin, Serbia. Main research areas: Public Relations, Organizational Culture and Knowledge Management.

Milena Nedeljković, PhD, University of Novi Sad, Faculty of Science, Department of Geography, Tourism and Hotel Management, Serbia. Main research areas: Organizational Psychology with a particular focus on job satisfaction and leadership.
} 
Das Ziel dieses Artikels ist es, die Beziehungen zwischen den GLOBE KulturDimensionen, der Arbeitszufriedenheit und dem Führer-Mitarbeiter-Verhältnis in serbischen Organisationen zu untersuchen. Einer der wichtigsten Aspekte dieser Studie war die Demonstration der Nützlichkeit der Anwendung allgemeiner Kulturtheorien bei der Untersuchung von Unternehmenskulturen. Die Stichprobe besteht aus 256 mittleren Führungskräften aus serbischen Organisationen. Die erzielten Ergebnisse zeigen, dass die OrganisationskulturDimensionen signifikante Prädiktoren für alle Facetten der Arbeitszufriedenheit sind. Zudem werden die Beziehungen zwischen den OrganisationskulturDimensionen und der Arbeitszufriedenheit durch die Führer-MitarbeiterVerhältnis-Variable moderiert. Unsere Ergebnisse können für Personalmanager in serbischen Organisationen von Interesse sein, ebenso wie für ausländische Investoren.

Keywords: Organizational culture, job satisfaction, LMX, Serbia.

\section{Introduction}

After the democratic political changes in many Eastern European countries, there has been a growing interest in understanding national culture, organizational culture and leadership processes in their organizations. In spite of that interest there is a lack of such an investigation in Serbian organizations. Feichtinger and Fink (1998) claim that cultural processes and features in transition countries which are usually attributed to the communist heritage are the result of collective culture shock. They have formulated a theory of "collective culture shock" and built their theory on the achievements of anthropologists and cross-cultural researchers. They used the definition and the symptoms of individual culture shock (a prolonged stress reaction to a new situation and milieu) which they describe as: "a well known and evidenced phenomenon. It describes the psychological and physical reactions of a person staying abroad. These reactions are the result of confrontation with a foreign culture. This collective culture shock influences management and business relations and causes problems". Among the former state-owned enterprises, the diffusion of modern organizational structures and management practices still seems to be limited even in the fast developing CEE societies, especially, where ownership lies in the hands of insiders or the state (Mikl-Horke 2004). The transfer of the knowledge of the market economy is proceeding quite slowly because of many cultural tensions and conflicts (Alas/Vadi, 2004).

During the Cold War, Serbia (like all the Yugoslav republics) had a specific position as a socialist country largely independent of the impact of the Sovietled communist bloc. Citizens were free to travel around the world and were in a position to encounter other political and economic systems. The Serbian system of workers' self-management had certain characteristics of participative 
management. Participation in the decision-making process had historical roots since large consultative bodies combined with a paternalistic leadership style (asking the opinion of others) was a dominant pattern of leadership in Serbian organizations in the long period of Serbian socialism. At the same time, the presence of certain elements of a market economy was one of the differences between the Serbian and communist Soviet-led bloc economies. Maybe, for this reason, the introduction of Western management cultural values in Serbian organizations did not cause such a high level of culture shock as it did in many countries from the former communist bloc.

Since job satisfaction is one of the predictors of many important organizational outcomes, including job performance (Judge et al. 2001), it is interesting to investigate the relationships between organizational culture dimensions and general or facet job satisfaction in Serbian organizations. Job satisfaction is one of the most researched phenomena (attitudes) in the literature on organizational behavior. By 1972, over 3,000 studies were published examining the antecedents and consequences of job satisfaction (Locke, 1976). In addition to this, there has been no indication that the study of job satisfaction has diminished in the 35 years since then. As such, Spector (1997) summarized that it is undoubtedly the most widely studied variable in I/O psychology. He further notes that job satisfaction is a central variable in many theories that deal with organizational phenomena, such as the nature of work, supervision, and the job environment. Research in the field has shown a correlation between job satisfaction and variables such as organizational commitment, absence, turnover, motivation and organizational culture. One approach for obtaining a measure of job satisfaction is to ask employees directly about their attitudes about their job, called a global scale, or some aspects of their job. Global scales ask the subject to combine his or her reactions to various aspects of their job in a single, integrated response. Conversely, facet scales are intended to cover each of the principal areas separately within the general satisfaction domain. Each facet is intended to be relatively homogeneous and distinctly different from the others. Rather than using measures of overall job satisfaction, one could focus on the relationship between the separate facet satisfaction score and some other organizational constructs. The examination of these facet conditions is often useful for a more careful examination of employee satisfaction with critical job factors. Traditional job satisfaction facets include: co-workers, pay, job conditions, supervision, nature of the work and benefits.

Many investigators have pointed out the connections between organizational culture and job satisfaction; the results depend on the dimensions of organizational culture chosen by the authors. The general conclusion is that some dimensions of organizational culture (for example, competitiveness, innovation, performance orientation, emphasis on rewards, social responsibility, stability and supportiveness) influence general job satisfaction significantly. 
Hofstede pointed out that all organizations are embedded within societal cultures, which are likely to have an ambient influence on the organizations embedded within them. He demonstrated the utility of applying more general cultural theory to the study of organizational cultures (Hofstede, 1980, 1991, 1998, 2001). Several decades ago, Hofstede also investigated cultural dimensions in some former Yugoslav republics (Slovenia, Croatia and Serbia) (Hofstede, 1980). According to his findings power distance (76) and uncertainty avoidance (88) indexes in these republics were very high and individualism (27) and masculinity indexes (21) were very low. After Hofstede's investigations of national culture dimensions, Serbia, like other former Yugoslav republics, went through a process of many political and economic changes, but there is a lack of research on how these changes have instigated changes in national and organizational cultural dimensions.

The appearance of the GLOBE project's main monograph (House et al., 2004) marked an important point in the development of Hofstede's doctrine. The GLOBE project was partly inspired by Hofstede's studies and was intended, among other things, as a corrective of Hofstede's model. While the GLOBE researchers fully accepted Hofstede's paradigm of constructing dimensions of national culture from variables that correlate across nations, they felt that some of his dimensions lacked face validity and they did not measure what was implied by their labels. GLOBE's work not only contributed to a better understanding and appreciation of Hofstede's work but also elucidated some previously murky points in cross-cultural research. Except for Slovenia (which was included in the GLOBE project) the GLOBE national and organizational cultural dimensions have not been studied in other former Yugoslav republics

In this paper, we investigate the level of GLOBE organizational cultural dimensions, the relationships between organizational cultural dimensions and facets job satisfaction, and the moderating effect of the leader-member exchange relation on them in Serbian organizations.

\section{Organizational culture, job satisfaction and LMX theory}

\section{Organizational culture}

Since organizational culture is connected with many organizational outcomes (House et al. 2004), including job satisfaction, there is a growing body of research related to organizational culture definitions and dimensions. Although there is no agreement on a single definition of organizational culture, it may be generally assumed that the concept of organizational culture tends to be defined as a set of basic assumptions, values, attitudes and norms of behavior shared within an organization and manifested through their members' perceptions, thoughts, feelings and behavior, as well as artifacts of both a material and nonmaterial nature (Deal/Kennedy, 1982; Lauzen /Dozier,1994: Wallack 1983; Schein 1985; Deshpande /Webster 1989; Kotter 1992, 1996; Conner 1992; 
Cummings/ Worley 2005). Schein (1985) maintains that culture exists at both the cognitive and emotional level, and he views behavior as a manifestation of culture.

Denison (1996) notes that a fundamental dilemma which is often faced in the literature on organizational culture is that theories tend to posit that individuals influence an organizational culture, and that they are also influenced by the organizational culture. While micro-level theories might equate organizational culture with an employee's perceptions of an organization, in order for those perceptions to be truly "organizational," they must be based on a common environmental stimulus present in the organization. Organizational cultures partly develop in response to stimuli that are experienced in common by organizational members. Sources of such concrete or common environmental elements include the leadership practices that are utilized throughout the organization, and become manifest in institutionalized structures and rules.

For researchers to understand culture they need to assess the broader cultural paradigm of a society within which the organization operates since this influences the manner in which the organization operates. The initial aim of the GLOBE (Global Leadership and Organizational Behaviour) research project was to develop societal and organizational measures of culture and leadership that are appropriate for use across cultures. GLOBE is a multi-phase, multi-method project initiated by Robert House in 1993. A detailed analysis on the relationship between Hofstede's and GLOBE's approaches to cultural dimensions is given in a paper by Minkov and Hofstede (2011).

Over 170 social scientists and management scholars from 62 countries in all major regions throughout the world were engaged in the study of the relationship between culture and leadership. The GLOBE team reached a consensus on the definition of the two central concepts: societal culture and leadership. The GLOBE project empirically established nine cultural dimensions that make it possible to capture the similarities and/or differences in norms, values, beliefs and practices among societies and organizations. For conceptual reasons GLOBE expanded the five Hofstede dimensions to nine. They maintained the labels Power Distance and Uncertainty Avoidance (but not necessarily their meaning). They split Collectivism into Institutional Collectivism and In-Group Collectivism, and Masculinity-Femininity into Assertiveness and Gender Egalitarianism. Long Term Orientation became Future Orientation. They added two more dimensions: Humane Orientation and Performance Orientation.

The nine GLOBE dimensions were covered by 78 survey questions, half of them asking subjects to describe their culture ('as is') and the other half to judge it ('as it should be'). GLOBE thus produced $9 \times 2=18$ culture scores for each country: nine dimensions 'as is' (practices) and nine 'should be' (values). 
The nine "as is" GLOBE cultural dimensions are:

Uncertainty Avoidance: The extent to which a society, organization, or group relies on social norms, rules, and procedures to alleviate unpredictability of future events.

Future oriented: The degree to which a collective encourages and rewards future-oriented behaviors such as planning and delaying gratification.

Power Distance: The degree to which members of a collective expect power to be distributed equally.

Collectivism I (Institutional): The degree to which organizational and societal institutional practices encourage and reward the collective distribution of resources and collective action.

Humane Orientation: The degree to which a collective encourages and rewards individuals for being fair, altruistic, generous, caring, and kind to others.

Performance Orientation: The degree to which a collective encourages and rewards group members for performance improvement.

Collectivism II (In-Group): The degree to which individuals express pride, loyalty, and cohesiveness in their organizations or families.

Gender Egalitarianism: The degree to which a collective minimizes gender inequality.

Assertiveness: The degree to which individuals are assertive, confrontational, and aggressive in their relationships with others.

The distinction between practices and values is relative. "For any specific case, any practice was at some point a value, an expectation, a model before it became an actual cultural product. Similarly, values are perpetuated through repetitive practices. In all languages, the word value expresses something worthy to follow, a goal, a stimulus, a model. Usually the values are about something important, and therefore are supposed to be followed. If they are not important (and not followed), they are not values. Perhaps this is why some authors believe that values express the effectiveness of most efficient individuals, such as leaders while practices express the average effectiveness of a society (organization)" (House et al. 2004).

Harry Triandis, one of the major figures in cross-cultural research, calls the GLOBE research project, in the foreword to the first GLOBE volume, "the Manhattan Project of the study of the relationship of culture to conceptions of leadership" (2004). There are a number of reasons for this assessment. GLOBE is the most comprehensive study to date that has empirically researched the relationship between culture and leader behavior in many societies, with many different quantitative and qualitative measures and methods, and in many different organizations. Perhaps, most importantly, and in the words of its 
principal researcher, Robert J. House, "[M]y final conclusion is that we are in a position to make a major contribution to the organizational behavior and leadership literature. To date more than $90 \%$ of the organizational behavior literature reflects U.S.-based research and theory. Hopefully GLOBE will be able to liberate organizational behavior from U.S. hegemony" (2004).

\section{Job satisfaction}

There are many definitions of job satisfaction. Locke (1976) defined job satisfaction as a pleasurable or positive emotional state resulting from the appraisal of one's job or job experiences. According to Spector (1997), job satisfaction is a person's evaluation of his or her job and work context i.e. an attitude reflecting how well people like or dislike their job (Spector 1997). In this paper, Spector's definition will be applied. Job satisfaction is viewed, as an attitude to the job either in its entirety (global satisfaction) or about particular aspects (facet satisfaction). The latter usually relates to several job facets, including the work itself, supervision, pay, promotion opportunities, and coworkers.

Because of the multi-dimensionality of job satisfaction/dissatisfaction it should be measured in terms of individual dimensions instead of a global construct. Measurement of individual dimensions of job satisfaction enables researchers to identify the environmental factors (for example, cultural variables) related to certain dimensions of job satisfaction.

Many papers have been published on the connections between organizational culture and job satisfaction. Lund (2003), adopting Cameron and Quinn's (1999) typology of cultures, identified a positive relationship between clan (emphasizing flexibility and internal focus) and adhocracy (emphasizing innovation, creativity, and adaptability) cultures and job satisfaction and a negative relationship between the market (stressing goal achievement and beating competition) and hierarchy (stressing efficiency and smooth functioning) cultures. Similarly, Silverthorne (2004) concluded that the greatest level of job satisfaction is more likely to be produced when the culture is supportive than when it is innovative or when it is bureaucratic. As for cultural dimensions, McKinnon et al. (2003) argue that respect for people, innovation, stability and aggressiveness had a positive effect on job satisfaction whereas Huang and $\mathrm{Wu}(2000)$ concluded that result orientation, professional features, and severe control and management increase employee job satisfaction. Platonova et al. (2006), examining hospital employees, found that employees who felt that the organization recognized their job performance were more likely to be satisfied. 


\section{Leader-member exchange theory}

LMX theory is a leadership theory that describes the quality of supervisorsubordinate relationships (Dansereau et al. 1975). LMX quality is important because it relates to employee satisfaction, promotions, performance ratings, OCBs, and communication behaviors (Gerstner/Day 1997; Liden et al. 1997). High-LMX (in-group) members share mutual trust, respect, reciprocal influence, loyalty, liking, and a sense of obligation with their leaders (Graen/Uhl-Bien 1995). Employees with high-LMX relationships have more opportunities to speak up, exchange information or ideas with their supervisors, and use more communication channels compared to those in low-LMX relationships (Fairhurst 1993). They have more communication exchanges with their supervisor and benefit from greater work support and supervisor responsiveness. In contrast, low-LMX (out-group) members have more formal and restricted relationships that are based on economic exchange and are characterized by low trust, low support, and few rewards. As a result, employees in low-LMX relationships have less access to their supervisors, fewer resources, and restricted information (Fairhurst/ Chandler 1989). By virtue of their position in the organizational hierarchy (linking pin), leaders enjoy the power to decide how to distribute valued resources and key opportunities among subordinates. This is necessary if the leader is to be able to treat subordinates in a differentiated manner (Dienesch, Liden, 1986). Green et al. (1995) report the amount of financial resources available within the organization to be positively related to LMX quality. This is because resource availability provides leaders with more discretion and latitude in terms of initiating a high quality relationship with subordinates.

There are many benefits of the high quality of the leader-member exchange relation on the organizational and individual levels (Scandura/Graen, 1984; Crouch/Yetton, 1988; Erdogan et al. 2006; Pellegrini/Scandura, 2006) including promotion, turnover, organizational commitment, job satisfaction, citizenship behaviors, willingness to contribute, performance, and trust in supervisors (Gerstner/Day, 1997; Liden et al. 1997; Graen et al. 1982; Stepina et al. 1991; Stringer 2006; Erdogan/Enders, 2007; Anseel/Lievens, 2007; Katrinli et al. 2010).

\section{Hypotheses}

Let the notations OCi and JSj mean:

OC1-uncertainty avoidance, OC2-future orientation, OC3-power distance, OC4collectivism 1 (institutional), OC5-human orientation, OC6-performance orientation, OC7-collectivism 2 (in-group collectivism), OC8-gender egalitarianism and OC9-assertiveness.

JS1 (satisfaction with pay), JS2 (satisfaction with promotion), JS3 (satisfaction with supervisor), JS4 (satisfaction with fringe benefits), JS5 (satisfaction with 
contingent reward), JS6 (satisfaction with operative procedure), JS7 (satisfaction with co-workers), JS8 (nature of work), JS9 (communication).

In this paper we shall investigate the following two general hypotheses:

H1-organizational culture dimensions significantly predict facets of job satisfaction

H2-LMX moderates the relationships between organizational culture dimensions and facets of job satisfaction.

$\mathrm{H} 1$ consists of 81 sub-hypotheses $\mathrm{H}(1, \mathrm{OCi}, \mathrm{JSj})(\mathrm{i}, \mathrm{j}=1,2,3,4,5,6,7,8,9)$ where sub-hypotheses $\mathrm{H}(1, \mathrm{OCi}, \mathrm{JSj})$ are defined in the following way:

$\mathrm{H}(1, \mathrm{OC}, \mathrm{JSj})$ - organizational culture dimension OCi predicts facet of job satisfaction JSj.

Hypothesis $\mathrm{H} 2$ consists of 81 sub-hypotheses $\mathrm{H}(2, \mathrm{OCi}, \mathrm{JSj})(\mathrm{i}, \mathrm{j}=1,2,3,4,5,6$, $7,8,9)$ where sub-hypotheses $\mathrm{H}(2, \mathrm{OC}, \mathrm{JSj})$ are defined in the following way:

$\mathrm{H}(2, \mathrm{OCi}, \mathrm{JSj})$ - LMX moderates the regression between organizational culture dimension OCi and facet of job satisfaction JSj.

\section{Method}

\section{Participants and data collection}

The sample consists of 256 middle managers from 131 organizations in Serbia (134 employees in the sample work in state organizations and 122 in private ones), in which more than 50 workers are employed. In smaller organizations in Serbia, there are frequently only several managers of the same level and an owner who is not a supervisor of managers in a standard sense. Namely, in many cases in such organizations, the owner has no previous experience in management and is not involved fully in the management process. Hence, we decided to choose organizations with more than 50 workers as our sample. The organizations studied are among Serbian's top 250 enterprises (related to financial performance-profit). All the middle managers in the sample have Bachelors and Masters Degrees, 136 of them are male and 120 female.

\section{Survey instruments}

Since the GLOBE project presents an additional, expanded model of cultural measures (Venaik/ Brewer 2008), we used the GLOBE project instrument for measuring organizational culture dimension "as is". The "should be" part of the instrument is used only in order to compare the values obtained from this part of the GLOBE instrument with the values from the "as is" part of the instrument. The GLOBE questionnaire has 34 items and the answers are measured on the 7point Likert scale. These instruments measure nine organizational culture dimensions: Performance Orientation, Future Orientation, Gender Egalitarianism, Assertiveness, Institutional Collectivism, In-Group Collectivism, 
Power Distance, Humane Orientation and Uncertainty Avoidance (House et al. 1999,2002 , 2004). Job satisfaction is measured by the Job Satisfaction Survey (Spector 1985), which has 36 items related to nine facets of job satisfaction: Pay, Promotion, Supervision, Fringe Benefits, Contingent Rewards, Operating Procedures, Co-workers, Nature of Work, and Communication. The answers are measured by the 6-point Likert scale. We used the LMX-7 instrument (Graen/Uhl-Bien, 1995) which has 7 items and answers were measured by the 5point Likert scale. Some of the questions were: How well does your leader recognize your potential? How well does your leader understand your job problems and needs? Regardless of how much formal authority your leader has built into his or her position, what are the chances that your leader would use his or her power to help you solve problems in your work? The LMX-7 instrument is one of the most commonly used instruments for the estimation of the quality of the OCi LMX relation (Hughes et al. 2010). The Cronbach alpha for all questionnaires was greater than 0.70 .

\section{Data analysis}

We used both a hierarchical regression analysis (Milin/Hadzic 2011) and the Chow test (Chow, 1960) in order to investigate the moderating effect of LMX on the regression between $\mathrm{JSj}$ (as a dependent variable) and OCi (as an independent variable), where $\mathrm{i}, \mathrm{j}=1,2,3,4,5,6,7,8,9$.

The hierarchical regression analysis consists of three steps. In the first step OCi is a predictor, in the second step OCi, LMX are predictors, and in the last step, an interactive variable LMX x OCi is added into the model. The moderating effect was tested by examining the change in the R-squared attributable to the interaction term. If the interaction term added at the final stage of the regression analysis produced a significant R-squared (i.e., significantly increased the amount of variance explained in the outcome variable) the LMX could be said to be a moderator of the relationship between $\mathrm{OCi}$ and $\mathrm{JSj}$.

The Chow test (Chow, 1960) is a method well known in econometrics. The method can be used to determine if two regression lines are different from one another. Significant interactions were analyzed by sub-grouping analysis, in which participants were split into appropriate groups. In this study, the moderator variable LMX is split at the median into two groups (low LMX and high LMX). After sub-grouping the subjects, the regression coefficients in the subgroups are compared in order to investigate the influence of the variable LMX on the regression equations between the predictor variables (OSi) and the criterion variables ( $\mathrm{JSj}$ ) for each subgroup. The F-ratio for the Chow test was calculated using the residual sum of squares of the regression equations. This permitted the rejection of the hypothesis of equality between the regression coefficients in regression equations for low and high LMX sub-samples. By the Chow test, the variable LMX is a moderator if the regression coefficients in the 
regression equations between the predictor and the criterion variables in the subsamples differed significantly.

\section{Descriptive statistics}

In Table 1, we give the results of the Descriptive Statistics (mean and standard deviations) for the "as is" dimension of the organizational culture, the facet of job satisfaction and the LMX and the abbreviations for these variables that we shall use later in the paper.

Table 1: Descriptive Statistics

\begin{tabular}{|c|c|c|c|c|}
\hline & Short name & $\mathrm{N}$ & Mean & SD \\
\hline Uncertainty Avoidance & OC1 & 256 & 3.8006 & 1.34038 \\
\hline Future Oriented & OC2 & 256 & 4.3080 & 1.63478 \\
\hline Power Distance & OC3 & 256 & 4.7946 & 1.42911 \\
\hline Collectivism 1 & OC4 & 256 & 3.8199 & 1.35587 \\
\hline Humane Orientation & OC5 & 256 & 4.2232 & 1.41515 \\
\hline Performance Orientation & OC6 & 256 & 3.9408 & 1.36935 \\
\hline Collectivism 2 & OC7 & 256 & 4.5634 & 1.18553 \\
\hline Gender Egalitarianism & OC8 & 256 & 2.9301 & 1.20891 \\
\hline Assertiveness & OC9 & 256 & 3.7656 & 1.09026 \\
\hline Pay & JS1 & 256 & 2.9107 & 1.32451 \\
\hline Promotion & JS2 & 256 & 3.3304 & 1.18838 \\
\hline Supervision & JS3 & 256 & 3.6641 & 1.29293 \\
\hline Fringe Benefits & JS4 & 256 & 2.9989 & 1.25291 \\
\hline Contingent Rewards & JS5 & 256 & 3.1529 & 1.35047 \\
\hline Operating Procedures & JS6 & 256 & 3.1830 & 1.04199 \\
\hline Coworkers & JS7 & 256 & 4.3571 & 1.13777 \\
\hline Nature of Work & JS8 & 256 & 4.4900 & 1.23553 \\
\hline Communication & JS9 & 256 & 3.9699 & 1.31775 \\
\hline LMX-7 & LMX & 256 & 3.1665 & .96876 \\
\hline Valid N (listwise) & & 256 & & \\
\hline
\end{tabular}

In Table 2 we give the average values of the "as is" and the "should be" dimensions of organizational culture in Serbia and for the overall GLOBE Data ("as is") (Javidan/House 2001). 
Table 2: Organizational culture dimensions

\begin{tabular}{|l|c|c|c|c|}
\hline \multirow{2}{*}{} & \multicolumn{2}{|c|}{ Serbia } & \multicolumn{2}{c|}{$\begin{array}{c}\text { Overall GLOBE Data } \\
\text { (As Is) } \\
\text { (Javidan, House, 2001) }\end{array}$} \\
\cline { 2 - 5 } & As Is & $\begin{array}{c}\text { Should } \\
\text { Be }\end{array}$ & $\begin{array}{c}\text { Highest } \\
\text { score }\end{array}$ & $\begin{array}{c}\text { Lowest } \\
\text { Score }\end{array}$ \\
\hline Uncertainty Avoidance & 3.80 & 5.21 & 5.37 & 2.88 \\
\hline Future Oriented & 4.31 & 5.99 & 5.07 & 2.88 \\
\hline Power Distance & 4.80 & 3.13 & 5.80 & 3.89 \\
\hline Collectivism 1 & 3.82 & 4.65 & 5.22 & 3.25 \\
\hline Humane Orientation & 4.22 & 5.50 & 5.12 & 3.18 \\
\hline Performance Orientation & 3.94 & 6.11 & 4.90 & 2.88 \\
\hline Collectivism 2 & 4.56 & 5.61 & 6.03 & 3.53 \\
\hline Gender Egalitarianism & 2.93 & 4.80 & 4.08 & 2.50 \\
\hline Assertiveness & 3.77 & 2.86 & 4.73 & 3.38 \\
\hline
\end{tabular}

\section{Correlation and regression analysis}

In Table 3 we give the Pearson coefficients of the correlation between OCi and $\mathrm{JSi}$, for all $\mathrm{i}=1,2,3,4,5,6,7,8,9$.

Table 3: Pearson coefficients of the correlation between organizational culture and job satisfaction

\begin{tabular}{|c|c|c|c|c|c|c|c|c|c|}
\hline & JS1 & JS2 & JS3 & JS4 & JS5 & JS6 & JS7 & JS8 & JS9 \\
\hline OC1 & $.328^{* *}$ & $.278^{* *}$ & $.260^{* *}$ & $.229^{* *}$ & $.229^{* *}$ & .037 & $.171^{*}$ & $.131^{*}$ & $.371^{* *}$ \\
\hline OC2 & $.511^{* *}$ & $.440^{* *}$ & $.412^{* *}$ & $.410^{* *}$ & $.470^{* *}$ & $-.208^{* *}$ & $.418^{* *}$ & $.311^{* *}$ & $.523^{* *}$ \\
\hline OC3 & $-.410^{* *}$ & $-.370^{* *}$ & $-.344^{* *}$ & $-.295^{* *}$ & $-.395^{* *}$ & $.179^{* *}$ & $-.259^{* *}$ & $-.219^{* *}$ & $-.433^{* *}$ \\
\hline OC4 & $.364^{* *}$ & $.343^{* *}$ & $.210^{* *}$ & $.241^{* *}$ & $.395^{* *}$ & -.055 & $.269^{* *}$ & $.163^{*}$ & $.314^{* *}$ \\
\hline OC5 & $.471^{* *}$ & $.441^{* *}$ & $.371^{* *}$ & $.335^{* *}$ & $.467^{* *}$ & $-.149^{*}$ & $.499^{* *}$ & $.359^{* *}$ & $.485^{* *}$ \\
\hline OC6 & $.626^{* *}$ & $.565^{* *}$ & $.492^{* *}$ & $.448^{* *}$ & $.627^{* *}$ & $-.172^{*}$ & $.448^{* *}$ & $.370^{* *}$ & $.608^{* *}$ \\
\hline OC7 & $.555^{* *}$ & $.480^{* *}$ & $.469^{* *}$ & $.465^{* *}$ & $.529^{* *}$ & $-.198^{* *}$ & $.477^{* *}$ & $.437^{* *}$ & $.570^{* *}$ \\
\hline OC8 & .066 & .054 & .110 & .127 & $.133^{*}$ & $-.132^{*}$ & .014 & .039 & $.162^{*}$ \\
\hline OC9 & -.014 & .041 & -.007 & -.074 & -.083 & -.042 & -.081 & -.025 & -.088 \\
\hline
\end{tabular}

${ }^{* *} p<0.01 ; *{ }^{*} p<0.05$ 
From the obtained results, we concluded that $\mathrm{H}(1, \mathrm{OCi}, \mathrm{JSj})$ is confirmed for:

1. $\quad \mathrm{i}=1$ and $\mathrm{j}=1,2,3,4,5,7,8,9$.

2. $\quad I=2$ and $j=1,2,3,4,5,6,7,8,9$.

3. $\quad \mathrm{i}=3$ and $\mathrm{j}=1,2,3,4,5,6,7,8,9$.

4. $\quad i=4$ and $j=1,2,3,4,5,6,7,8,9$.

5. $\quad i=5$ and $j=1,2,3,4,5,6,7,8,9$.

6. $\quad \mathrm{i}=6$ and $\mathrm{j}=1,2,3,4,5,6,7,8,9$.

7. $\quad i=7$ and $j=1,2,3,4,5,6,7,8,9$.

8. $\quad \mathrm{i}=8$ and $\mathrm{j}=5,6,9$.

By the multiple regression method we also investigated the contribution of all the independent variables OCi to the total R-square in the regression with JSj as a dependent variable. The dimensions of the organizational culture are the best predictors for communication and contingent reward $\left(\mathrm{R}^{2}=0.440\right)$. This tells us that $44 \%$ of the variation in communication and contingent reward can be predicted using GLOBE dimensions of organizational culture. For predictions of pay, promotion, supervision, fringe benefit, contingent reward, operating procedure, coworkers, nature of work and communication, $\mathrm{R}^{2}$ and $\mathrm{F}$ change are respectively- $\mathrm{R}^{2}=0.425$ ( $\mathrm{F}$ change-17.598, $\mathrm{p}<0,001$ ), $\mathrm{R}^{2}=0.344$ ( $\mathrm{F}$ change-12.482, $\mathrm{p}<0,001), \mathrm{R}^{2}=0.281 \quad(\mathrm{~F}$ change-9,315, $\mathrm{p}<0,001), \mathrm{R}^{2}=0.266$ ( $\mathrm{F}$ change-8.630, $\mathrm{p}<0,001$ ), $\mathrm{R}^{2}=0.440$ (F change-18.705, $\mathrm{p}<0,001$ ), $\mathrm{R}^{2}=0.100$ (F change-2.643, $\mathrm{p}<0,006), \mathrm{R}^{2}=0.310$ (F change-10,698, $\left.\mathrm{p}<0,001\right), \mathrm{R}^{2}=0.296$ ( $\mathrm{F}$ change-6.168, $\mathrm{p}<0,001), \mathrm{R}^{2}=0.440$ (F change-18,711, $\left.\mathrm{p}<0,001\right)$.

The variable OC6 (performance orientation) has the greatest influence on variables JS1 (pay), JS2 (promotion), JS3 (supervisor), JS5 (contingent reward) and JS9 (communication). The variable OC7 (in-group collectivism) has the greatest influence on variables JS4 (fringe benefits) and JS8 (nature of work). The variable $\mathrm{OC} 1$ (uncertainty avoidance) has the greatest influence on variable JS6 (operative procedure), and variable OC5 (human orientation) has the greatest influence on variable JS7 (co-workers). 
Figure 1: Multiple regression

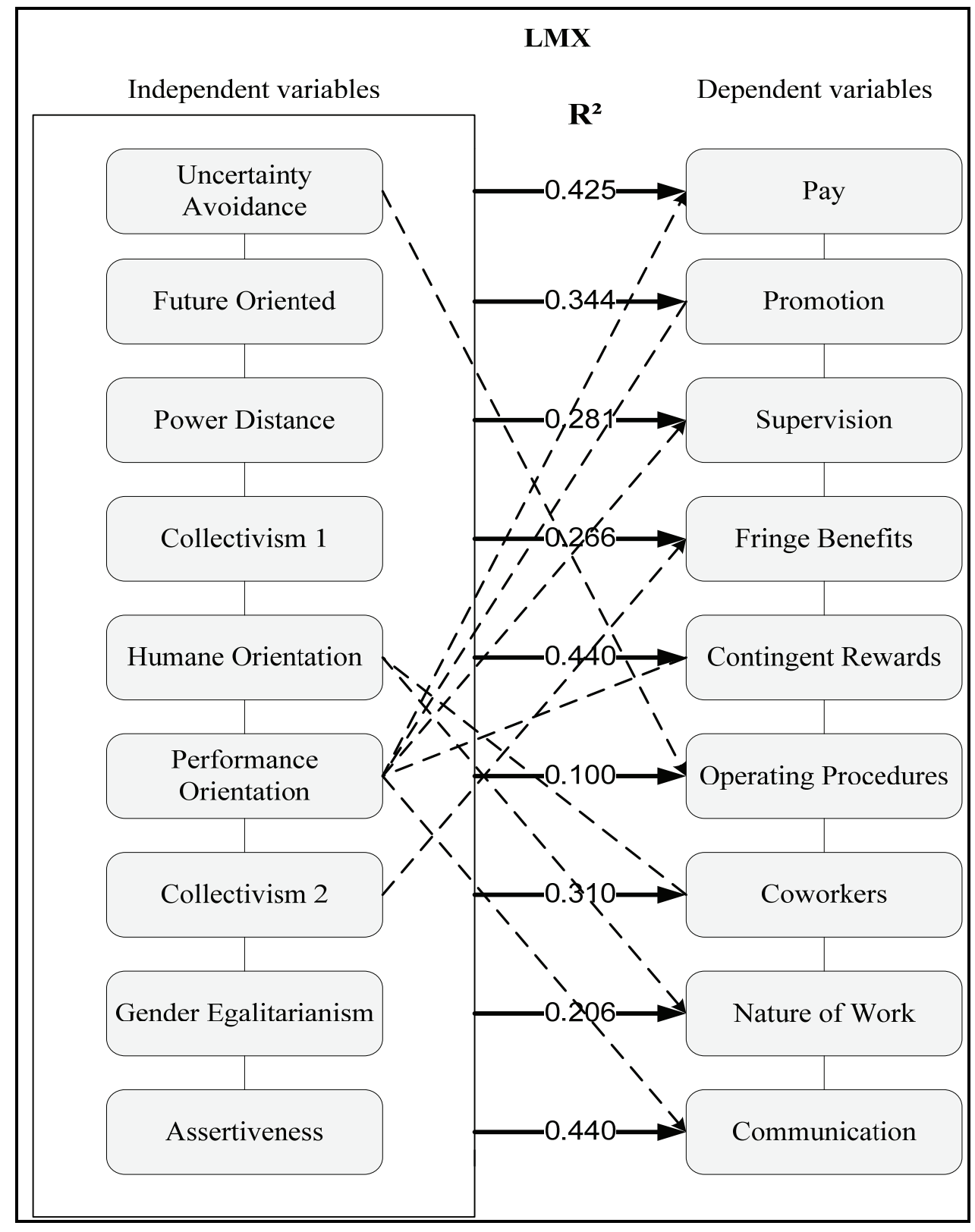

In Table 4 we give the Pearson coefficients of the correlation between OCi and JSj in low LMX (LLMX) and high LMX (HLMX) sub-samples for all i,j =1, 2, $3,4,5,6,7,8,9$.

The results of the Chow test for the difference between regression coefficients in the regression equations between OCi and JSi in the LLMX and HLMX subsamples are presented in Table 5. 


\begin{tabular}{|c|c|c|c|c|c|c|c|c|c|c|}
\hline \multirow{2}{*}{$\stackrel{\partial}{2}$} & $\sum_{\Xi}^{X}$ & $\begin{array}{l}* \\
\stackrel{*}{*} \\
\stackrel{\infty}{+}\end{array}$ & $\begin{array}{l}\text { * } \\
n \\
n \\
n\end{array}$ & 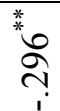 & ?ָ & 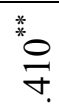 & $\begin{array}{l}\text { * } \\
\text { ? } \\
\text { n? }\end{array}$ & $\begin{array}{l}\text { * } \\
\text { in } \\
n\end{array}$ & $\overrightarrow{0}$ & $\begin{array}{l}\infty \\
\infty \\
0\end{array}$ \\
\hline & $\sum_{3}^{\bar{x}}$ & 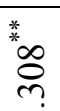 & $\stackrel{*}{\stackrel{*}{*}} \underset{+}{\stackrel{*}{+}}$ & $\stackrel{*}{*} \underset{\sim}{\stackrel{*}{*}}$ & $\stackrel{\infty}{0}$ & $\stackrel{*}{\stackrel{*}{*}}$ & 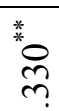 & 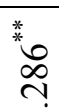 & $\begin{array}{l}\text { * } \\
\text { ?유 } \\
\text { ? }\end{array}$ & $\begin{array}{l}\text { * } \\
\stackrel{*}{*}\end{array}$ \\
\hline \multirow{2}{*}{$\stackrel{\infty}{\mathscr{n}}$} & $\sum_{\exists}^{\swarrow}$ & $\stackrel{g}{ \pm}$ & $\stackrel{*}{*} \underset{f}{\stackrel{*}{*}}$ & $\stackrel{n}{i}$ & $\stackrel{*}{\stackrel{*}{ָ}}$ & ? & 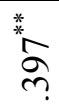 & $\begin{array}{l}* \\
\text { *n } \\
i n \\
7\end{array}$ & ठิ & $\widehat{\widehat{o}}$ \\
\hline & $\sum_{\exists}^{凶}$ & $\hat{\sigma}_{i}^{n}$ & $\cong$ & $\hat{g}_{i}$ & $\underset{i}{0}$ & $\stackrel{n}{\leftrightharpoons}$ & $\stackrel{\Xi}{\Xi}$ & 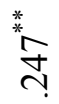 & $\stackrel{\infty}{0}$ & 命 \\
\hline \multirow{2}{*}{$\hat{\approx}$} & $\sum_{\exists}^{凶}$ & $\stackrel{*}{\sigma}$ & $\stackrel{*}{*} \frac{*}{7}$ & $\underset{0}{0}$ & $\stackrel{*}{*}$ & $\begin{array}{l}* \\
* \\
\stackrel{*}{*}\end{array}$ & : & * & ఠి & $\hat{g}_{i}$ \\
\hline & 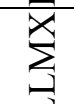 & $\stackrel{0}{0}$ & $\stackrel{*}{*}$ & $\stackrel{n}{ \pm}$ & $\stackrel{\varrho}{=}$ & $\stackrel{*}{*}{ }^{*}$ & $\stackrel{g}{+}$ & $\stackrel{*}{n}$ & ฮิ & $\underline{6}$ \\
\hline \multirow{2}{*}{$\stackrel{\mho}{\mathscr{2}}$} & $\sum_{\exists}^{\chi}$ & $\stackrel{ \pm}{\beth}$ & $\tilde{o}_{i}^{n}$ & है & f & ஜ & $\hat{\mathrm{o}}$ & $\begin{array}{l}0 \\
\infty \\
0\end{array}$ & $\hat{g}_{i}$ & $\stackrel{*}{\stackrel{*}{a}}$ \\
\hline & $\sum_{j}^{\bar{x}}$ & $\stackrel{?}{\text { T }}$ & $\stackrel{*}{\stackrel{*}{*}}$ & $\stackrel{*}{2} \stackrel{2}{?}$ & $\overbrace{i}^{0}$ & $\stackrel{*}{*}$ & gे & $\stackrel{尺}{i}$ & $\underset{\sigma}{0}$ & $\hat{0}$ \\
\hline \multirow{2}{*}{$\tilde{n}$} & $\sum_{\exists}^{凶}$ & $\stackrel{0}{=}$ & $\stackrel{*}{*}$ & $\stackrel{\text { * }}{\text { * }}$ & $\stackrel{*}{*} \stackrel{*}{\sim}$ & $\stackrel{\text { 荌 }}{\stackrel{+}{+}}$ & 悉 & $\stackrel{*}{n}$ & $\stackrel{\infty}{5}$ & ô. \\
\hline & $\sum_{a}^{\bar{\lambda}}$ & $\stackrel{0}{n}$ & $\begin{array}{l}\text { 粪 } \\
\text { nn }\end{array}$ & $\stackrel{*}{*}$ & 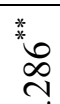 & $\stackrel{\overbrace{}}{\longrightarrow}$ & 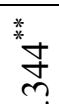 & $\stackrel{*}{\stackrel{\circ}{a}}$ & $\stackrel{{ }^{*}}{\stackrel{N}{N}}$ & $\stackrel{m}{\rightleftharpoons}$ \\
\hline \multirow{2}{*}{ 节 } & $\sum_{\exists}^{\grave{\lambda}}$ & $\sqrt{6}$ & $\stackrel{*}{*}$ & $\stackrel{\text { * }}{\stackrel{*}{*}}$ & $\stackrel{*}{*}$ & 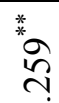 & $\stackrel{*}{\nabla}$ & $\stackrel{*}{*} \stackrel{*}{*}$ & $\stackrel{+}{=}$ & 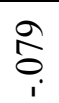 \\
\hline & $\sum_{J}^{\swarrow}$ & $\tilde{n}$ & "ै. & $\stackrel{n}{n}$ & 8̊ & $\infty$ & $\stackrel{*}{*}$ & $\begin{array}{l}* \\
* \\
\stackrel{0}{*} \\
\stackrel{0}{*}\end{array}$ & I & 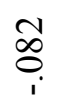 \\
\hline \multirow{2}{*}{$\underline{\tilde{n}}$} & $\sum_{\exists}^{\infty}$ & $\stackrel{n}{\infty}$ & $\stackrel{*}{*} \vec{\pi}$ & 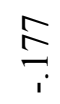 & $\stackrel{\infty}{0}$ & $\stackrel{*}{*} \underset{?}{n}$ & $\stackrel{*}{\frac{*}{*}}$ & $\stackrel{*}{*}$ & $\tilde{ర}_{i}$ & 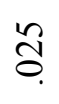 \\
\hline & $\sum_{-}^{\swarrow}$ & 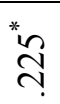 & $\stackrel{\text { * }}{\stackrel{*}{n}}$ & $\stackrel{*}{2} \stackrel{2}{\varrho}$ & $\hat{8}$ & $\stackrel{N}{0}$ & * & $\stackrel{*}{\stackrel{*}{\Upsilon}}$ & 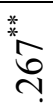 & $\tilde{n}_{i}^{n}$ \\
\hline \multirow{2}{*}{$\underset{\approx}{\sim}$} & $\sum_{\mid}^{X}$ & $\stackrel{{ }^{*} \infty}{\infty}$ & 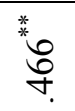 & * & $\begin{array}{l}{ }^{*} \\
{ }^{\infty} \\
n \\
n \\
m\end{array}$ & ले & $\begin{array}{l}\text { * } \\
\text { ñ } \\
n\end{array}$ & $\stackrel{*}{*} \stackrel{+}{*}$ & $\overbrace{i}^{+}$ & bे \\
\hline & $\sum_{\exists}^{x}$ & 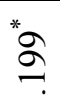 & "* & " & $\stackrel{n}{ \pm}$ & ํㅗำ & $\stackrel{*}{*}$ & $\stackrel{\overbrace{}}{\longrightarrow}$ & $\stackrel{\infty}{N}$ & $\frac{\infty}{0}$ \\
\hline \multirow{2}{*}{$\underline{\tilde{D}}$} & $\sum_{\exists}^{\infty}$ & $\begin{array}{l}* \\
\text { *o } \\
\stackrel{\infty}{\infty}\end{array}$ & 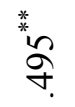 & $\stackrel{*}{*}$ & $\frac{*}{m}$ & $\stackrel{*}{*}$ & $\stackrel{*}{*}$ & $\stackrel{*}{n}$ & ฮิ & $\underset{i}{\sigma_{i}}$ \\
\hline & $\sum_{\exists}^{\bar{ג}}$ & $\stackrel{*}{\sigma}$ & $\stackrel{*}{\stackrel{*}{f}}$ & $\stackrel{*}{*}$ & $\stackrel{*}{\oplus}$ & 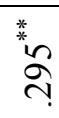 & 苾 & ๙े & \&̊ & $\stackrel{0}{0}$ \\
\hline & $\frac{x}{z}$ & $\bar{\circlearrowright}$ & ঠે & రુ & ঠூ & ¿ֶ & రి & $\hat{0}$ & రొ & తి \\
\hline
\end{tabular}




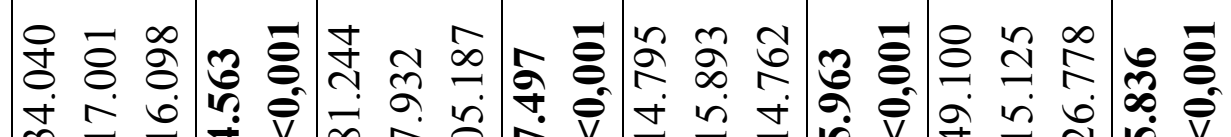

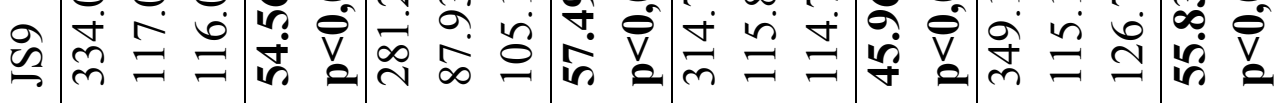

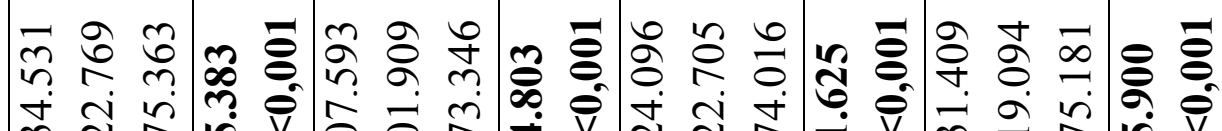

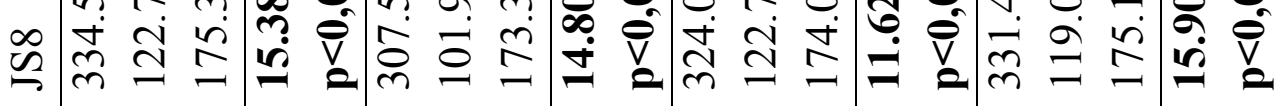

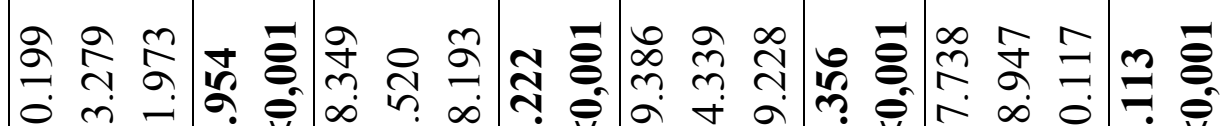

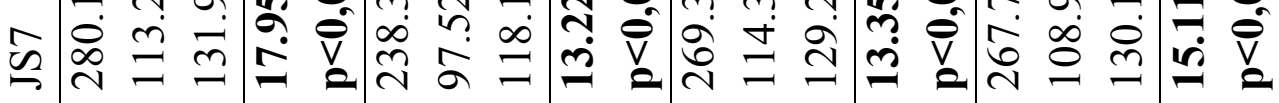

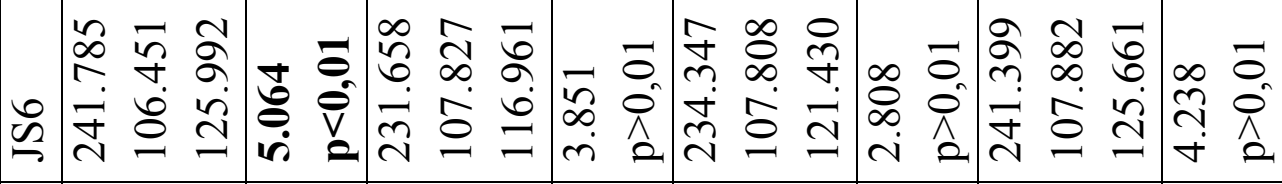

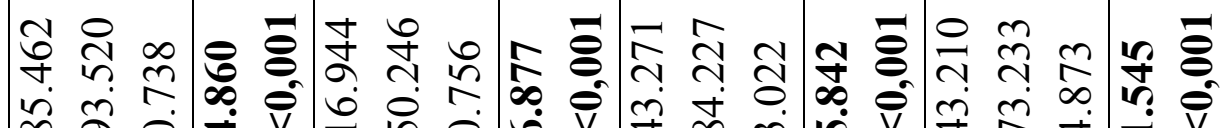

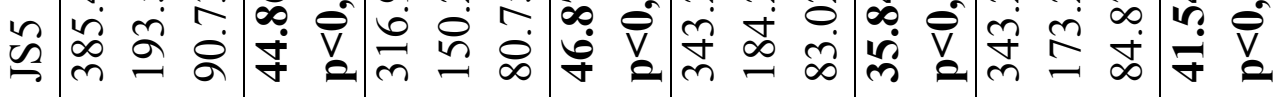

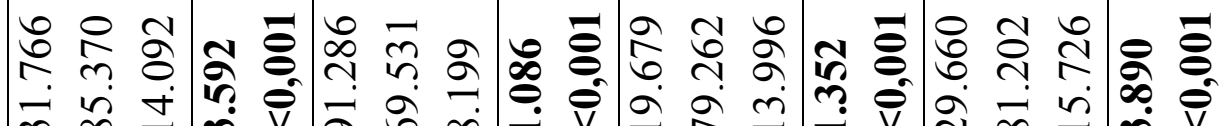

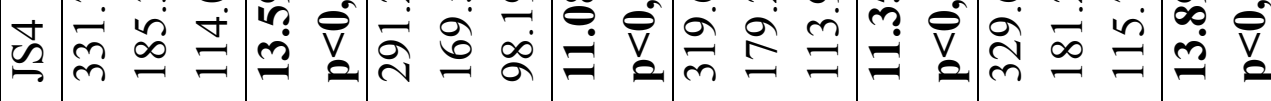

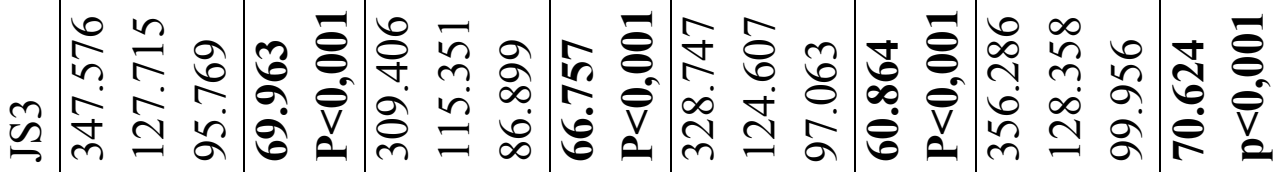

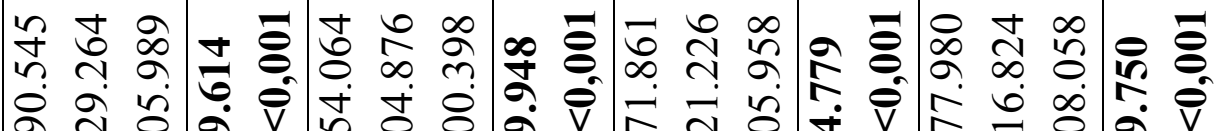

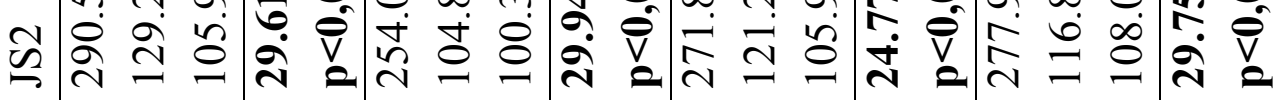

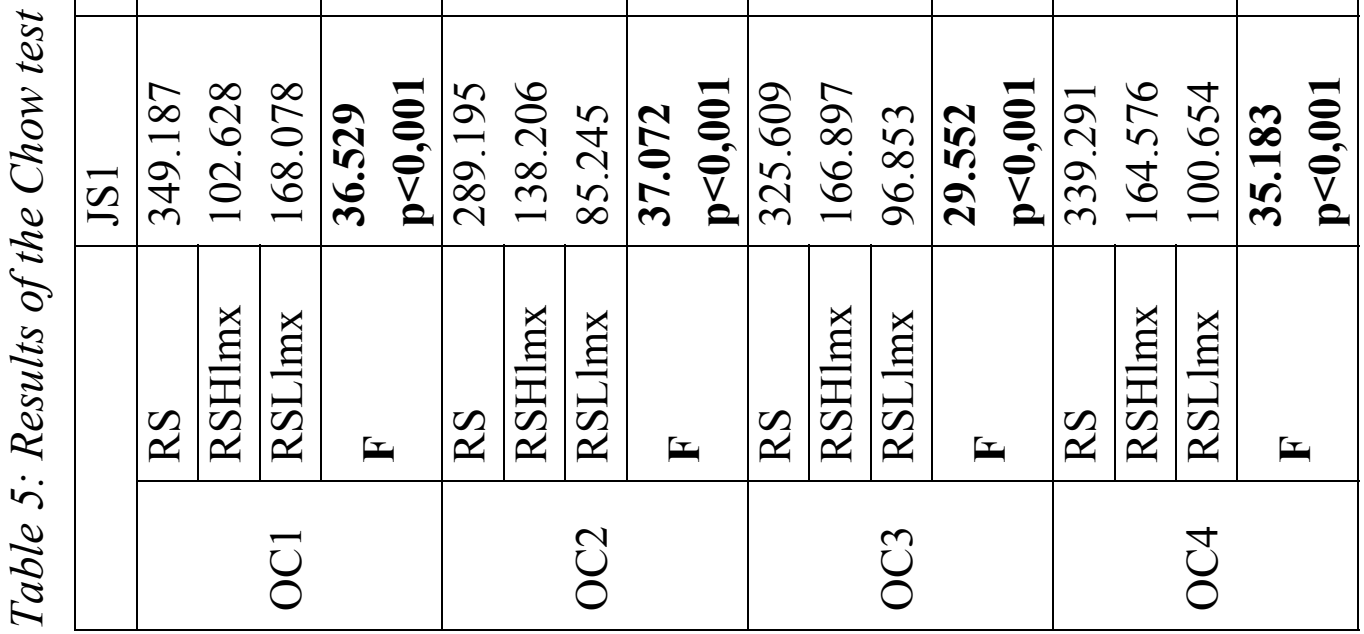




\begin{tabular}{|c|c|c|c|c|c|c|c|c|c|c|c|c|}
\hline 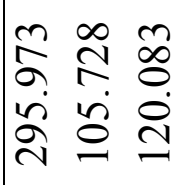 & 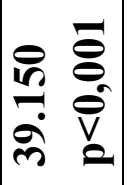 & 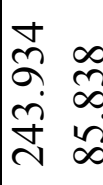 & 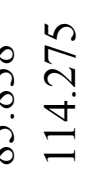 & 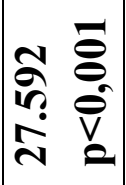 & 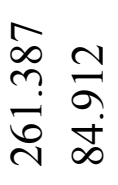 & 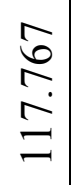 & $\begin{array}{ll}\hat{N} & \bar{\theta} \\
\hat{\sigma} & \hat{\theta} \\
\dot{0} & \bar{v} \\
\text { ñ } & 2\end{array}$ & 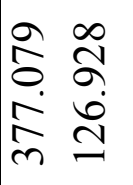 & $\begin{array}{l}n \\
n \\
n \\
=\end{array}$ & 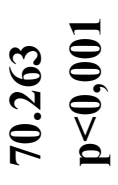 & 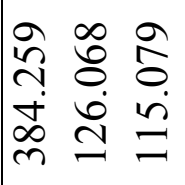 & $\begin{array}{ll}0 & \bar{\theta} \\
\hat{N} & \hat{\sigma} \\
\dot{1} & \bar{v} \\
N & 0\end{array}$ \\
\hline 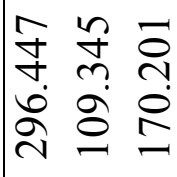 & \begin{tabular}{ll} 
& $\bar{\theta}$ \\
$\infty$ & $\hat{\theta}$ \\
\hdashline & $\bar{v}$ \\
$r$ & 2
\end{tabular} \mid & 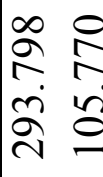 & 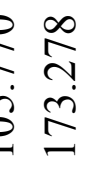 & 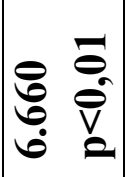 & \begin{tabular}{ll}
$\stackrel{\infty}{*}$ & $a$ \\
\multirow{v}{*}{} & $n$ \\
$\stackrel{n}{*}$ & $\Omega$ \\
& $\sigma$
\end{tabular} & $\begin{array}{l}\mathfrak{b} \\
\infty \\
\dot{\sigma} \\
-\end{array}$ & 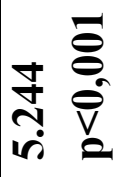 & 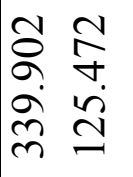 & 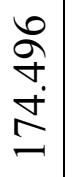 & 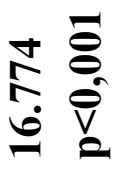 & 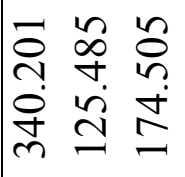 & \begin{tabular}{ll}
0 & $\overline{8}$ \\
$\infty$ & 0 \\
$\infty$ & $\hat{\theta}$ \\
0 & $\bar{v}$ \\
\hdashline & 0
\end{tabular} \\
\hline 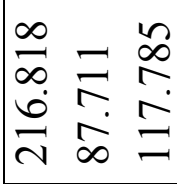 & 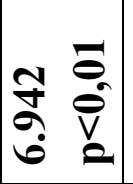 & $\begin{array}{ll}0 & \\
0 & \bar{\sigma} \\
\infty & 5 \\
0 & j \\
& \infty\end{array}$ & $\begin{array}{l}\hat{\sigma} \\
0 \\
0 \\
0 \\
0 \\
0\end{array}$ & 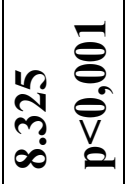 & 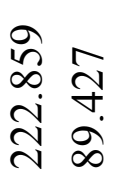 & 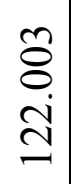 & $\begin{array}{ll} & \bar{\theta} \\
\bar{\theta} & \hat{\theta} \\
\infty & \bar{v} \\
0 & 2\end{array}$ & 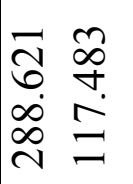 & 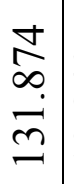 & 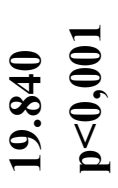 & 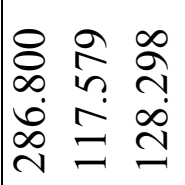 & $\begin{array}{ll}\overrightarrow{\hat{N}} & \bar{\theta} \\
\hat{\theta} & \hat{\hat{\theta}} \\
\dot{\hat{\theta}} & \bar{v}\end{array}$ \\
\hline 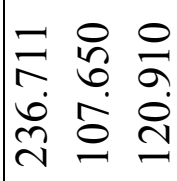 & $\begin{array}{cc}\Re & 0 \\
\sigma & 0 \\
\dot{\gamma} & \hat{1}\end{array} \mid$ & 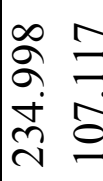 & 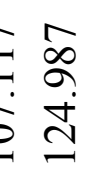 & \begin{tabular}{ll}
$\overline{1}$ & 0 \\
$\hat{n}$ & 0 \\
\hdashline & $\hat{0}$ \\
- & 0
\end{tabular} & 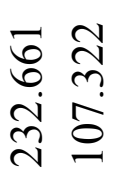 & $\begin{array}{l}\stackrel{a}{ \pm} \\
\underset{ป}{\simeq}\end{array}$ & $\begin{array}{ll}0 & 0 \\
0 & 0 \\
0 & \hat{0} \\
-1 & 0\end{array}$ & 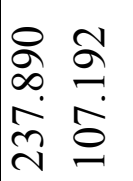 & $\begin{array}{l}\text { } \\
\text { ปे } \\
\text { ป̂ }\end{array}$ & $\begin{array}{ll}\hat{n} & \overline{0} \\
\sim & 0 \\
\forall & \hat{0}\end{array}$ & 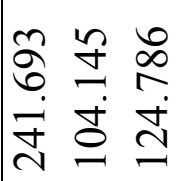 & \begin{tabular}{ll} 
& $\bar{\theta}$ \\
\multirow{2}{*}{} & $\hat{\theta}$ \\
$\hat{\theta}$ & $\bar{v}$ \\
$\sim$ & 2
\end{tabular} \\
\hline $\begin{array}{lll}\bar{N} & 0 & 0 \\
\infty & 0 & 0 \\
\sim & \tilde{N} & \infty \\
\bar{m} & n & 0\end{array}$ & $\begin{array}{cc}n & \bar{\theta} \\
\infty & 0 \\
\infty & \hat{\theta} \\
\infty & \bar{v} \\
\infty & 2\end{array}$ & $\begin{array}{ll}n & n \\
0 & n \\
\infty & n \\
0 & 0 \\
\vdots & 1 \\
d & 1\end{array}$ & 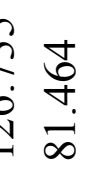 & $\begin{array}{cc} & \bar{\theta} \\
8 & \theta \\
\dot{\theta} & \hat{\theta} \\
\dot{v} & \bar{V} \\
\ddot{v} & 2\end{array}$ & 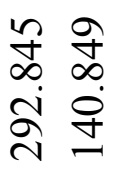 & $\begin{array}{l}\widehat{N} \\
\infty \\
\infty \\
\infty\end{array}$ & 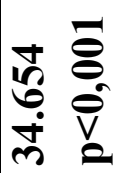 & 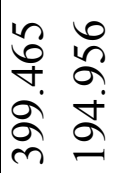 & $\begin{array}{l}\infty \\
\infty \\
\infty \\
\infty \\
\infty\end{array}$ & 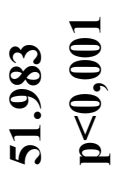 & 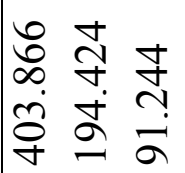 & $\begin{array}{ll}+ & \bar{\theta} \\
m & \hat{\sigma} \\
\dot{\sigma} & \bar{v} \\
\hat{n} & 0\end{array}$ \\
\hline 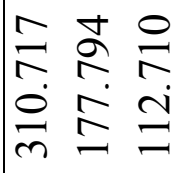 & $\begin{array}{ll} & \bar{\theta} \\
\hat{\sigma} & \hat{\theta} \\
0 & \bar{v} \\
\infty & 2\end{array} \mid$ & 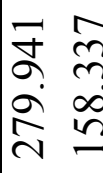 & $\begin{array}{l}\hat{6} \\
\vdots \\
0 \\
0\end{array}$ & 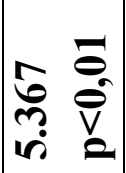 & 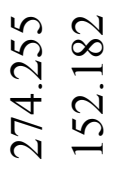 & $\begin{array}{l}n \\
\tilde{\sigma} \\
\hat{0} \\
-\end{array}$ & 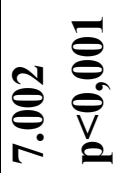 & 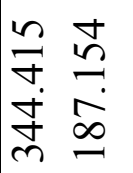 & 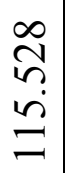 & 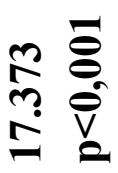 & 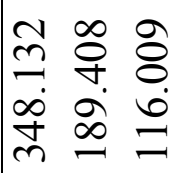 & 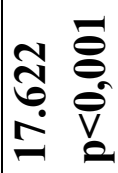 \\
\hline 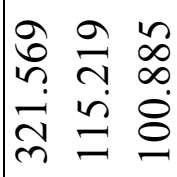 & $\begin{array}{ll}v & \bar{\theta} \\
\hat{\sigma} & \bar{\sigma} \\
\dot{\sigma} & \bar{v} \\
\sigma & 2\end{array}$ & 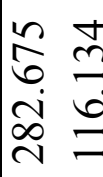 & 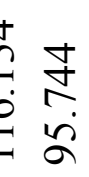 & 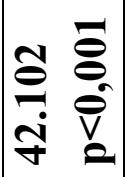 & 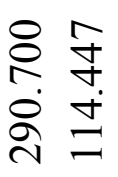 & $\begin{array}{l}0 \\
0 \\
0 \\
0 \\
0\end{array}$ & 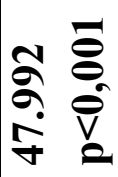 & $\begin{array}{ll}\hat{N} & \infty \\
& n \\
\infty & \infty \\
0 & \infty \\
0 & 1\end{array}$ & $\frac{o}{}$ & $\begin{array}{ll}\varkappa & \bar{\theta} \\
& \hat{\theta} \\
& \bar{v}\end{array}$ & 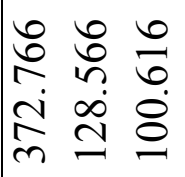 & $\begin{array}{ll}\theta & \overline{8} \\
\dot{1} & \sigma \\
0 & \hat{\theta} \\
\infty & 0\end{array}$ \\
\hline 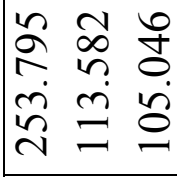 & 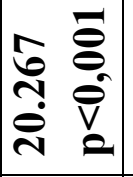 & 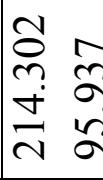 & $\begin{array}{l}\infty \\
\hat{n} \\
\hat{n} \\
\hat{n} \\
\hat{n}\end{array}$ & 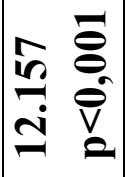 & 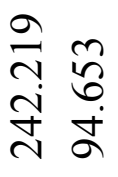 & $\begin{array}{c}\stackrel{\vartheta}{ナ} \\
\stackrel{\infty}{0} \\
\stackrel{0}{ }\end{array}$ & 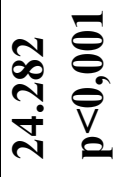 & $\begin{array}{ll}\hat{\sigma} & \hat{\sigma} \\
\sigma & \infty \\
m & \dot{n} \\
m & =\end{array}$ & \begin{tabular}{l}
$\infty$ \\
\multirow{+}{*}{} \\
$\infty$ \\
0
\end{tabular} & 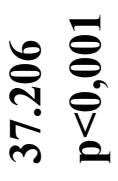 & 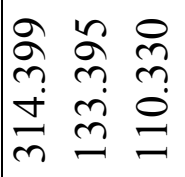 & 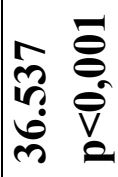 \\
\hline 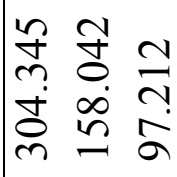 & $\begin{array}{ll}\infty & \bar{\theta} \\
\tilde{N} & \hat{\sigma} \\
\sim & \bar{v} \\
\dot{\sim} & \overline{2}\end{array}$ & 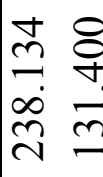 & $\begin{array}{l}n \\
\vdots \\
i \\
i \\
i \\
\infty \\
\infty \\
\infty \\
\infty\end{array}$ & 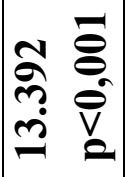 & 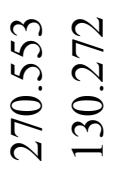 & 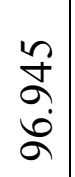 & 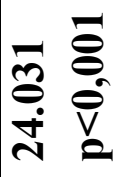 & $\begin{array}{ll}n & 0 \\
& \Omega \\
2 & i \\
\infty & \infty \\
n & 0\end{array}$ & 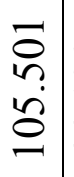 & 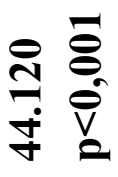 & 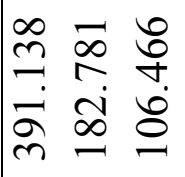 & $\begin{array}{ll}n & \overline{8} \\
\infty & \hat{\sigma} \\
r & \hat{\theta} \\
\dot{\nabla} & \bar{v}\end{array}$ \\
\hline 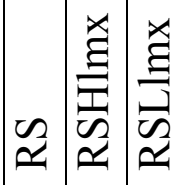 & Ex & 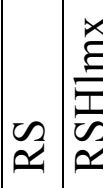 & 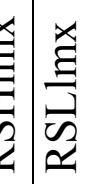 & $E$ & $\underset{\widetilde{E}}{\mathscr{E}}$ & 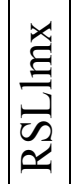 & {$[x$} & 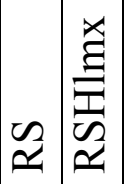 & 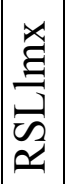 & {$[1$} & 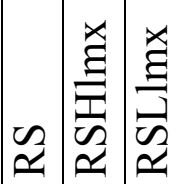 & {$[1$} \\
\hline 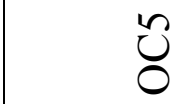 & & & 0 & & & 0 & & & 0 & & & \\
\hline
\end{tabular}


Because of the limits on the number of words in the paper, the details of the hierarchical regression analysis have not been included. They can be obtained from the authors on request. In Table 6 , only the results related to R-square and F-change statistics that support $\mathrm{H}(2, \mathrm{OCi}, \mathrm{JSj})$ are presented.

Table 6: Hierarchical regression analysis ( $R$ square and $F$-change)

\begin{tabular}{|l|l|l|}
\hline & R square & F-change \\
\hline $\begin{array}{l}\text { Dependent variable: Promotion } \\
\text { Independent variable: Future } \\
\text { Oriented }\end{array}$ & 0,377 & $4,222$. \\
\hline $\begin{array}{l}\text { Dependent variable: Contingent } \\
\text { reward } \\
\text { Independent variable: Future Oriented }\end{array}$ & 0,517 & $12,143$. \\
\hline $\begin{array}{l}\text { Dependent variable: Communication } \\
\text { Independent variable: Future Oriented }\end{array}$ & 0,546 & $5,273$. \\
\hline $\begin{array}{l}\text { Dependent variable: Contingent } \\
\text { reward } \\
\text { Independent variable:Collectivism 1 }\end{array}$ & 0,495 & $5,498$. \\
\hline $\begin{array}{l}\text { Dependent variable: Nature of of } \\
\text { work } \\
\text { Independent variable: Collectivism 1 }\end{array}$ & 0,244 & 4,500 \\
\hline $\begin{array}{l}\text { Dependent variable: Supervisor } \\
\text { Independent variable: Humane } \\
\text { oriented }\end{array}$ & 0,580 & 6,555 \\
\hline $\begin{array}{l}\text { Dependent variable: Operative } \\
\text { procedure: } \\
\text { Independent variable: Humane } \\
\text { oriented }\end{array}$ & 0,077 & 4,094 \\
\hline $\begin{array}{l}\text { Dependent variable: Promotion } \\
\text { Independent variable: Performance } \\
\text { oriented }\end{array}$ & 0,411 & 3,643 \\
\hline $\begin{array}{l}\text { Dependent variable: Supervisor } \\
\text { Independent variable: Performance } \\
\text { oriented }\end{array}$ & 0,578 & 12,073 \\
\hline $\begin{array}{l}\text { Dependent variable. Fringe benefit: } \\
\text { Independent variable: Performance } \\
\text { oriented }\end{array}$ & 0,236 & 3,184 \\
\hline $\begin{array}{l}\text { Dependent variable: Promotion } \\
\text { Independent variable: Collectivism 2 }\end{array}$ & 0,382 & 7,430 \\
\hline $\begin{array}{l}\text { Dependent variable: Fringe benefit } \\
\text { Independent variable: Collectivism 2 }\end{array}$ & 0,253 & 0,528 \\
\hline $\begin{array}{l}\text { Dependent variable: Contingernt } \\
\text { reward } \\
\text { Independent variable: Collectivism 2 }\end{array}$ & 0,510 & \\
\hline $\begin{array}{l}\text { Dependent variable: Communication } \\
\text { Independent variable: Assertiveness }\end{array}$ & 0,482 & \\
\hline
\end{tabular}


In the general sample, all the regression coefficients in the regression equations between $\mathrm{OC} 1$ and $\mathrm{JSj}$, for every $\mathrm{j}$ except for $\mathrm{j}=6$, are significantly positive. Hence, the increase of the perception of uncertainty avoidance is accompanied by the increase of satisfaction with all aspects of the job.

The Chow test supports $\mathrm{H}(2, \mathrm{OC} 1, \mathrm{JS} 1)$. In the sub-sample with high values of the LMX regression coefficient between $\mathrm{OC} 1$ and JS1 are statistically greater than in the sub-sample with the low values of LMX. The value of LMX.the Fstatistics, obtained by using the Chow test, is 36.529 , which is greater than the critical value $F$ (2.252) (which is less than 4.71 for $p=0.01$ ).

The Chow test supports $\mathrm{H}(2$, OC1, JS2). In the sub-sample with low values of the LMX regression, the coefficient between $\mathrm{OC} 1$ and JS2 is statistically greater than in the sub-sample with high values of LMX. The value of the F-statistics, obtained by using the Chow test, is 29.614 , which is greater than the critical value $F(2.252)$ for $\mathrm{p}=0.01$.

The Chow test supports $\mathrm{H}(2, \mathrm{OC} 1, \mathrm{JS} 3)$. In the sub-sample of low LMX, the regression coefficient between $\mathrm{OC} 1$ and JS3 is statistically greater than the corresponding coefficient in the sub-sample of high LMX. The value of Fstatistics obtained by the Chow test is 69.963 which is greater than the critical value for $\mathrm{F}$ (2.252) statistics.

The Chow test supports H(2, OC1, JS7). The regression coefficient between the independent variable $\mathrm{OC} 1$ and the dependent variable JS7 is, in the sub-sample LLMX, statistically greater than in the sub-sample HLMX. The F value by the Chow test is 17.954. Hence, the LMX moderates the relation between OC1 and JS7.

The Chow test supports $\mathrm{H}(2, \mathrm{OC} 1$, JS9). The regression coefficient between the independent variable $\mathrm{OC} 1$ and the dependent variable JS9 in the sub-sample LLMX is statistically greater than in the sub-sample HLMX. The F value by the Chow test is 54.563. Hence, LMX moderates the relation between OC1 and JS9. Hierarchical regression analysis does not support the moderator effect of LMX on the regression between OC1 and JSi.

In the general sample all the regression coefficients between the dependent variable $\mathrm{OC} 2$ and the independent variable $\mathrm{JSj}$, except for $\mathrm{j}=6$, are significantly positive, but for JS6 they are negative.

The Chow test supports $\mathrm{H}(2, \mathrm{OC} 2, \mathrm{JSj})$ for $\mathrm{j}=1,2,3,4,5,7,8$, 9. In the HLMX sub-sample, regression coefficients between independent variable $\mathrm{OC} 2$ and independent variables JS1 $(\mathrm{F}=37.072)$, JS2 $(\mathrm{F}=29.948)$, JS3 $(\mathrm{F}=66.757)$, JS4 $(\mathrm{F}=11.086), \quad J S 5 \quad(\mathrm{~F}=46.877), \quad J S 7 \quad(\mathrm{~F}=13.222), \quad J S 8 \quad(\mathrm{~F}=14.803)$ and JS9 $(\mathrm{F}=57.497)$ are positive and statistically greater than the corresponding coefficients in the LLMX sub-sample. Hierarchical regression analysis supports the moderator effect of LMX on the regression between OC2 and JS2, JS5 and JS9. 
In the general sample, all the regression coefficients between independent variable OC3 and dependent variables $\mathrm{JSj}$ are negative and significantly different from zero, except for $\mathrm{j}=6$ (where the corresponding coefficient is significantly positive).

The Chow test supports H(2, OC3, JS2). In the HLMX sub-sample, the increase of the perception of power distance is followed by a faster decrease of satisfaction with promotion than in the LLMX sub-sample $(\mathrm{F}=24.779)$. Hierarchical regression analysis does not support the moderator effect of the LMX on the regression between $\mathrm{OC} 3$ and $\mathrm{JSj}$.

In the general sample, all the regression coefficients between $\mathrm{OC} 4$ and $\mathrm{JSj}, \mathrm{j}=1$, $2,3,4,5,7,8,9$ are significantly positive. The regression coefficient between OC4 and JS6 is negative, but not significantly.

Applying the Chow test, we obtain that LMX moderates the regressions between OC4 and $\mathrm{JSj}, \mathrm{j}=1,2,3,4,5,7,8,9$; i.e. $\mathrm{H}(2, \mathrm{OC} 4, \mathrm{JSj})$ is proved for $\mathrm{j}=1,2,3,4$, $5,7,8,9$. In the HLMX sub-sample, the increase of the variable OC4 is followed by the faster increase of the variables JS1 $(\mathrm{F}=35.183)$, JS2 $(\mathrm{F}=29.750)$, JS3 ( $\mathrm{F}=70.624), \quad J S 4 \quad(\mathrm{~F}=13.890), \quad J S 5 \quad(\mathrm{~F}=41.545), \quad J S 7 \quad(\mathrm{~F}=15.113)$, JS8 $(\mathrm{F}=15.900)$ and JS9 $(\mathrm{F}=55.836)$, than is the case in the LLMX sub-sample. Hierarchical regression analysis supports the moderator effect of LMX on the regressions between OC3 and JS1, JS5 and JS8.

In the general sample, regression coefficients between the independent variable OC5 and the dependent variables JSj are significantly positive, except for $\mathrm{j}=6$, which is significantly negative.

Using the Chow test we obtain that LMX moderates the regressions between OC5 and $\mathrm{JSj}$ for $\mathrm{j}=1,2,3,4,5,7,8,9$; i.e. $\mathrm{H}(2, \mathrm{OC} 5, \mathrm{JSj})$ is proved for $\mathrm{j}=1,2,3$, $4,5,7,8,9$. In the HLMX sub-sample the increase of the variable OC5 is followed by the faster increase of the variables JS1 $(\mathrm{F}=24.233)$, JS2 $(\mathrm{F}=0.267)$, JS3 $(\mathrm{F}=61.492)$, JS4 ( $\mathrm{F}=8.767)$, JS5 $(\mathrm{F}=38.897)$, JS7 $(\mathrm{F}=6.942)$, JS8 $(\mathrm{F}=7.618)$ and JS9 $(\mathrm{F}=7.618)$, than in the LLMX sub-sample. In the LLMX sub-sample, the regression coefficient between OC5 and JS6 is significantly negative, and in the sub-sample, the HLMX is non-significantly positive. Hierarchical regression analysis supports the moderator effect of the LMX on the regressions between OC5 and JS3 and JS6.

In the general sub-sample all the regression coefficients in the regression equations between OC6 and JSi are significantly positive, except for $i=6$ which is significantly negative.

By the Chow test we obtain that LMX moderates the relation between OC6 and JS1 $(\mathrm{F}=13.392), \quad J S 2 \quad(\mathrm{~F}=12.157), \quad J S 3 \quad(\mathrm{~F}=42.102), \quad J S 5 \quad(\mathrm{~F}=23.400), \quad J S 7$ $(\mathrm{F}=8.325)$, JS8 $(\mathrm{F}=6.660)$, JS9 $(\mathrm{F}=27.592)$; i.e. $\mathrm{H}(2, \mathrm{OC} 6, \mathrm{JSj})$ is proved for $\mathrm{j}=1$, $2,3,5,7,8,9$. In the HLMX sub-sample, the increase of OC6 is followed by the faster increase of the variables $\mathrm{JSj}, \mathrm{j}=1,2,3,5,7,8,9$ than in the LLMX sub- 
sample. In the LLMX sub-sample, the regression coefficient between OC6 and JS6 is significantly negative, and in the HLMX sub-sample, it is nonsignificantly positive. Hierarchical regression analysis supports the moderator effect of the LMX on the regressions between OC6 and JS2, JS3, JS4 i JS9.

In the general sample, all the regression coefficients between OC7 and JSi are significantly positive except for $\mathrm{i}=6$ which is significantly negative.

By the Chow test we obtain that LMX moderates the relations between $\mathrm{OC} 7$ and $\mathrm{JSj}, \mathrm{j}=1,2,3,4,5,7,8$, 9; i.e. $\mathrm{H}(2, \mathrm{OC} 6, \mathrm{JSj})$ is proved for $\mathrm{j}=1,2,3,4,5,7,8,9$. In the HLMX sub-sample, the increase of OC7 is followed by the faster increase of the variables JS1( $\mathrm{F}=24.031)$, JS2 $(\mathrm{F}=24.031), \mathrm{JS} 3(\mathrm{~F}=47.992)$, JS4 $(\mathrm{F}=7.002)$, JS5 $(\mathrm{F}=34.654)$, JS7 $(\mathrm{F}=6.811)$, JS8 $(\mathrm{F}=36.497)$, JS9 $(\mathrm{F}=36.497)$, than in the sub-sample LLMX. Hierarchical regression analysis supports the moderator effect of the LMX on the regressions between OC7 and JS2, JS4 and JS5.

By the Chow method we obtained that the LMX moderates the relations between OC8 and JS3 $(\mathrm{F}=82.763)$. JS5 $(\mathrm{F}=82.763)$ and JS9 $(\mathrm{F}=82.763)$ i.e. $\mathrm{H}(2, \mathrm{OC} 8, \mathrm{JSj})$ is proved for $\mathrm{j}=3,5$, 9. In the LLMX sub-sample, the corresponding coefficients are significantly positive, which is not the case in the HMLX sub-sample. Hierarchical regression analysis does not support the moderator effect of the LMX on the regressions between OC8 and JSi, where $\mathrm{i}=1,2,3,4,5,6,7,8,9$.

Hierarchical regression analysis supports the moderator effect of the LMX on the regressions between OC9 and JS9, and the same result is supported by the Chow method $(\mathrm{F}=74.776)$.

\section{Discussion}

Comparing the perceived and expected GLOBE organizational culture indices (Table 2) we found the so called "pendulum effect" i.e. a substantial contrast between "as is" and "should be" indices (Bakacsi, 1999). Bakacsi found such an effect in a Hungarian sample and he posed the following hypotheses related to countries in the transition:

- The more a country (or an organization) perceives itself as a high power distance culture, the more it wants to change itself into a low power distance culture.

- The more a country (or an organization) perceives uncertainty, the more it strives to reduce it.

- The more a country (or an organization) has an individualistic culture, the more its members want to belong to a more collectivistic culture.

- The more a country (or an organization) perceives itself living in the present the more it wants to develop a longer future orientation. 
- The more people in a country (or an organization) tend to have assertive, dominant behavior in social relationships, the more they want to reduce this aggressiveness.

- The less a culture tends to encourage and reward individuals group members for performance improvement and excellence, the more it strives to change this pattern as a normative expectation.

- The less a culture tends to encourage and reward individuals for being fair, altruistic, generous, caring and kind to others the more it strives to change this pattern as a normative expectation.

\section{Uncertainty avoidance}

We obtain that the "as is" value of organizational uncertainty avoidance is 3.80 , and that "should be" is 5.21. These results are similar to the Hofstede conclusion on the uncertainty avoidance index being very high in the former Yugoslav republics, which can be partly explained by the fact that in the long period of socialism almost all aspects of job satisfaction were very predictable (pay, promotion...). It seems that middle managers in Serbian organizations are not yet ready to accept a lower level of uncertainty avoidance as a part of their organizational cultures.

Organizations in countries that are high on uncertainty avoidance show limited use of performance-based pay, and the salary of middle managers partly depends on the decisions of their leaders, which may be based to some extent on the quality of their relationship with their subordinates.

Many organizations in Serbia, especially those that are state owned, have a hierarchical organizational structure and the possibility for the promotion of middle managers is related not only to their performances but also to their age and educational level. Despite the fact that the possibility for promotion of middle managers does not depend much on the quality of their performance, a high level of LMX enhances a high level of expectation for promotion. When these expectations are not fulfilled, middle managers are disappointed and their level of satisfaction with promotion decreases. Under a high level of uncertainty avoidance, middle- managers in the low LMX group are aware of organizational realities, and that the possibility for promotion is also dependent on political factors outside the organization; the support of the leader is only one of the relations which may influence promotion.

At first glance, we obtain an unexpected result that, in the sub-sample of low LMX, the regression coefficient between OC1 and JS3 is statistically greater than the corresponding coefficient in the sub-sample of high LMX. Under conditions of higher uncertainty avoidance, a high LMX relationship may also involve a transactional (i.e. calculative) component, which may render trust to be vulnerable even in high-quality exchange relationships (Uhl-Bien, 2007). 
Since one of the items in the questionnaire related to satisfaction with the supervisor was the question about the competence of the supervisor, the abovementioned calculative component of the LMX relationship may be connected with the perception of the low level of the leader's competence. This reasoning is consistent with the underlying logic of LMX that subordinates are motivated to develop a high quality LMX with their supervisors only if they believe they will receive something of value in exchange. If they do not value the extra amount of support given by supervisors (for example, support for a higher level of autonomy because of the low level of the leader's competence), then the need for a high level of LMX is more likely to be low.

We obtained that the LMX moderates the relation between OC1 and JS7. A leader who is in a high quality LMX relationship with his or her subordinates, the middle managers, may help them to establish high quality relationships with key people in their social network, especially with their subordinates. Since employees' direct leaders are often in charge of administrating and allocating discretionary rewards, support, and resources, it is likely that the relationship between the leader and the employee plays an important role in influencing employee's perceived organizational support (Settoon et al. 1996; Wayne et al. 1997). Middle managers in the high LMX sub-sample can serve as a role model for their subordinates, who trust their managers because of the support they receive. Under a high level of uncertainty avoidance, co-workers of the middle managers in the sub-sample of HLMX are ready to work in accordance to their suggestions in order to fulfill the expectations of their supervisor, the middle manager. This may increase the level of satisfaction of the middle managers with their co-workers.

We obtained that the LMX moderates the relation between OC1 and JS9. Under higher uncertainty avoidance and the turbulent events of the past decade and ongoing challenges, employees in organizations in Serbia have a high need for information about the position of the organization on the financial market. Middle managers in the high LMX sub-sample expect to obtain necessary information from their leaders because of support for them, which may enhance their trust in the leader. However, leaders, especially in state owned organizations, are not ready, in many cases, to exchange bad information with their subordinates. Namely, the image of the organization may influence the authority of leaders among employees. These situations may cause the decreased level of satisfaction with communication among all employees and especially among middle managers.

\section{Future orientation}

The cultural dimension termed "future orientation" defined by House et al. (1999) refers to the extent to which members of a society or an organization believe that their current actions will influence their future performance, focus 
on investment in their future, believe in planning and look far into the future for assessing the effects of their current actions. Scholarly research has found a positive relationship between high future orientation and leadership behaviors such as strategic planning, entrepreneurship, decision-making, corruption, visioning, social responsibility, performance, development, and support of subordinates. In a highly future oriented organizational culture, human resource management practices are characterized by training and development, compensation and rewards, retention strategies, and organizational communication (Papalexandris/Panayotopoulou 2004; Zhao, 2006). In an organization with a high level of future orientation, the job tasks of middle managers become less formalized and mechanistic and they may be more motivated for entrepreneurial activities.

In the last fifteen years, Serbia has had quite serious economic and political problems and during that time the management in many organizations was short-term oriented i.e. one of managers' main tasks was to ensure the survival of the organization. Most of the employees were not really interested in developing new products since they were not oriented to entrepreneurship. We see from our research that the "should be" future orientation dimension is quite high, 5.99 and that middle managers are fully aware of the importance of a high level of future orientation. The increase of the future orientation value can be interpreted as the readiness for change. Middle managers do not expect immediate increase in pay and benefits and are ready to invest in research to achieve a good market position. Middle managers in Serbia are aware of the need for strategic entrepreneurship to create competitive advantages by using entrepreneurial initiatives to pursue opportunities and to transform existing organizations by redeploying resources and renewing strategic capabilities.

Since, in the general sample, all the regression coefficients between the dependent variable $\mathrm{OC} 2$ and the independent variable $\mathrm{JSj}$, except for $\mathrm{j}=6$, are significantly positive, and for JS6 it is negative, the increase of the perception of future orientation is followed by an increased satisfaction with many aspects of the job except for operative procedures. A high level of future orientation may cause the need for more planning and higher diversity of operative procedures, which may, in turn, cause a decrease in satisfaction with operative procedures.

By the Chow test, we concluded that in the HLMX sub-sample, the regression coefficients between the independent variable $\mathrm{OC} 2$ and the independent variables JS1, JS2, JS3, JS4, JS5, JS7, JS8 and JS9 are positive and statistically greater than corresponding coefficients in the LLMX sub-sample. A high value of LMX can help middle managers to obtain a greater amount of different resources from their leaders, which may help them to be more innovative and entrepreneurially oriented and also more risk oriented than middle managers in the low LMX sub-sample. Hence, the increase of the future orientation of middle managers in HLMX is followed by the faster increase of satisfaction 
with pay, promotion, supervisor, fringe benefits, contingent reward, co-workers, nature of work and communication, than in LLMX.

\section{Power distance}

Organizations that are high in power distance tend to expect obedience toward superiors and clearly distinguish between those with status and power and those without (House et al. 2004). In higher power distance organizations, power is seen as providing organizational order and role stability; information is controlled and only a few people have access to resources and pay, promotions and rewards, dependent mostly on the status of the employees. Low power distance organizations prefer participative relations, equal rights, and the use of legitimate rather than coercive power (House et al. 2004).

In our general sample, the average value of power distance ("as is") is 4.80 and the corresponding "as it should be" average value is 3.13, perhaps as a reaction to a high level of "as is" power distance in the long period under communism. It is quite understandable that middle managers in all countries seem to prefer lower levels of power distance than actually experienced. A high level of "as is" power distance and a low level of "should be" power distance seems to indicate a preference of Serbian middle managers for a more egalitarian approach to status in organizations and a desire for less privilege for middle manager supervisors.

Since, in the general sample, all the regression coefficients between the independent variable OC3 and the dependent variables JSj are negative and significantly different from zero, except for $\mathrm{j}=6$ (the corresponding coefficient is significantly positive), the increase of the perception of power distance is followed by a decrease of satisfaction with all aspects of job satisfaction, except for operative procedure. Clear operative procedures without much flexibility (under conditions of higher power distance perceptions) can help middle managers to avoid certain conflict situations, which may happen under conditions of flexibility and ambiguity of operative procedures.

From the statistical results, we concluded that in the HLMX sub-sample, the increase of the perception of power distance is followed by a faster decrease of satisfaction with promotion than in the LLMX sub-sample. It may be that under conditions of the perception of higher power distance, the support of the supervisor, which is the case in the high LMX sub-sample, produces a higher level of expectation with middle managers that they will be promoted than in the case of the LLMX sub-sample. In Serbian organizations, especially in those which are state owned, the possibility to be promoted to a high level in the managerial hierarchy depends not only on the decision of top managers but also on political authorities in the broader community, which is unfortunately a long tradition in Serbian organizations since the period of socialism. 
In the HLMX sub-sample, the increase of the perception of power distance is not followed by a significant decrease of satisfaction with the supervisor, which is not the case in the LLMX sub-sample. The increase of power distance in the low LMX sub-sample produces a cumulative negative effect on the level of satisfaction with the supervisor. The increase of the perception of power distance in the HLMX sub-sample is followed by a faster decrease of satisfaction with pay, fringe benefits, contingent reward, co-workers, nature of work and communications than in the LLMX sub-sample. This can be partially explained, as in the case of promotion, by the high level of expectation of middle managers produced by the support of their supervisors.

\section{Institutional collectivism}

Organizations with a high level of institutional collectivism practices encourage and reward the collective distribution of resources and collective action; predominantly as a member of a lifelong group or organization. In organizations with low institutional collectivism, the focus is on individual achievements and rewards.

In the general sample, the average value of "as is" institutional collectivism is 3.82 , and the average value of "should be" institutional collectivism is 4.65.

A high level of "should be" institutional collectivism (OC4) means that middle managers in Serbia consider that personal goals are of less importance than common organizational goals. It is obvious that the high value of "should be" institutional collectivism has its roots in the long period of socialism, during which the principle of egalitarianism was highly valued. Hence, the increased perception of institutional collectivism is followed by increased satisfaction with all aspects of the job, except for satisfaction with operative procedure.

We obtained that the LMX moderates the regressions between OC4 and $\mathrm{JSj}, \mathrm{j}=1$, $2,3,4,5,7,8,9$. Under the condition of the increase of OC4, middle managers in the HLMX sub-group, in accordance with the social exchange theory, feel obliged to be highly engaged in making good relationships with peers (first of all to establish good communication channels with their subordinates), and to set moderate personal goals related to pay, benefits and promotion. They take into consideration the objective power of the Serbian economy, the global economic crisis and the fact that many employees in their organizations have much lower pay and fringe benefits than they have.

\section{Humane orientation}

Since the human orientation dimension of organizational culture is defined as the degree to which an organization encourages and rewards individuals for being fair, altruistic, friendly, generous, caring, and kind to others, members of organizations in a high human orientation organizational culture are responsible for promoting the well-being of others. A high value placed on the humane 
orientation organizational cultural dimension is positively associated with the global leadership dimension of the "humane oriented type" i.e. such a type of leadership which reflects supportive and considerate relationships with subordinates. In our sample the average value of OC5 "as is" is 4.22 and the average value of OC5 "should be" is 5.50 .

Since in the general sample the regression coefficients between the independent variable OC5 and the dependent variables $\mathrm{JSj}$ are significantly positive, except for $\mathrm{j}=6$, which is significantly negative, the increase of perception of human orientation is followed by the increased satisfaction with all aspects of the job, except for satisfaction with operative procedure.

Using the Chow test, we obtained that LMX moderates the regressions between OC5 and JSj for all $\mathrm{j}=1,2,3,4,5,7,8$, 9; i.e. in the HLMX sub-sample the increase of the variable OC5 is followed by the faster increase of variables JS1, JS2, JS3, JS4, JS5, JS7, JS8 and JS9 than in the LLMX sub-sample. In the LLMX sub-sample, the regression coefficient between OC5 and JS6 is significantly negative, and in the HLMX sub-sample, it is non-significantly positive. Hence, a high level of LMX corresponds well, in some sense, with a high level of the human orientation organizational culture dimension, which partly explains the results obtained above. As we mentioned earlier, the leader can serve as a role model for middle managers. According to Bandura's social learning theory (1977), individuals learn by attending to and observing the behavior of important role models. An important part of the work of middle managers is to motivate and empower the employees of their working group. A high level of LMX can stimulate middle managers to engage in these, which may produce their increased satisfaction with the nature of their work. Furthermore, a high level of LMX, under the increased perception of the human orientation, enhances the freedom of middle managers to be actively engaged in the process of communication with their supervisor, in order to obtain important information about the position of the organization. Such a situation may increase the satisfaction of middle managers with communication in the organization.

\section{Performance orientation}

As we already mentioned, the average value of OC6 ("as is") in our sample is 4.22 and the average value of OC6 ("should be") is 5.50. Hence, employees in Serbia have a high level of the "should be" performance orientation dimension of organizational culture. A high value placed on performance orientation culture was also found to be significantly and positively associated with participative leadership. This reflects the degree to which managers involve others in making and implementing decisions (House et al. 2004).

A high level of LMX between middle managers and their leaders enhances joint efforts in making decisions, which may help in creating trust between these two parts of the management structure. Trust may help in understanding and 
accepting organizational changes, which is important for increasing the motivation for the performance of the entire staff. The organizations studied are among the most successful organizations in Serbia and the middle management is "rewarded" for successfully implementing performance oriented organizational culture by a high level of satisfaction with their co-workers, contingent rewards, communication and the nature of work.

\section{In-group collectivism}

Organizational in-group collectivism is, as mentioned above, the extent to which members of an organization take pride in and have loyalty to membership in the organization in which they are employed and are engaged in creating good relationships with the members of their working group. The average value of "as is" organizational in-group collectivism is 4.56 and of "should be" 5.61. This was to be expected, since the bond of family (and organization) has an unbreakable connection to the development of Serbian society. Through tradition and with different forms of the family, the family bond has constituted the first and strongest societal group through which the individual develops his personal identity. The connections between the employees and their organization were quite strong during the long period of socialism. Most of the employees worked in the same organization for all of their working lives. Hence, the increase of the value of $\mathrm{OC} 7$ is followed by an increased satisfaction with almost all (except JS6) aspects of the job.

If the LMX is low, the leader behaves in a manner inconsistent with the perceivers' (middle managers') expectations related to group cohesion. In the sub-sample of middle managers in which the level of LMX is high, leader behavior corresponds well to an increased level of organizational in-group collectivism. Hence, an increase of OC7 in the sub-sample of high LMX helps middle managers to be more motivated for their managerial duties, including the establishment of good relationships with their subordinates, which may result in higher performance and increase their satisfaction with pay, promotion, fringe benefits, contingent reward, the nature of work and communication.

\section{Gender egalitarianism}

Companies operating in a more gender-egalitarian organizational culture encourage tolerance for the diversity of ideas and roles regardless of gender. In our sample, the average value of OC8 "as is" is 2.93 , and "should be" is 4.80 . In reality, contrary to publicly proclaimed principles of gender egalitarianism, the situation in the studied organizations is quite different. In the general sample, regression coefficients between OC8 and JS5, JS6 and JS9 are significantly different from zero. The regression coefficients between OC8 and JS5 and JS9 are positive, and for JS6 negative. In our sample the increase of OC8 is followed by the increase of JS5 and JS9. More than $47 \%$ of employees are women and the increase of OC8 enhances the increased perception of the lack of gender 
barriers, which may produce the increased satisfaction with contingent reward and communication.

By the Chow method, we obtained that the LMX strongly moderates the relations between OC8 and JS3, JS5 and JS9. In the LLMX sub-sample, the corresponding coefficients are significantly positive, which is not the case in the HMLX sub-sample. One of the possible explanations is that under conditions of increased OC8 and high values of LMX (in the HMLX sub-sample) the expectations of middle managers (especially women) in respect to many aspects of their work are greater than reality. This situation may cause decreased satisfaction with many aspects of their job.

\section{Assertiveness}

In highly assertive countries like the United States, competition between individuals and groups is encouraged. Managers may set up incentives that reward the best idea, even if they are contrary to established practices. People in less assertive countries, like Sweden, New Zealand and Serbia, prefer harmony in relationships and emphasize loyalty and solidarity. The average value of OC9 "as is" in our sample is 3.77 , and "should be" is 2.86 . Hence, Serbian middle managers value a very low level of assertiveness.

Statistical analysis supported the moderator effect of LMX on the regressions between OC9 and JS9. Perhaps the increase of OC9 in the LLMX sub-sample employees produces an additional perception of the lack of harmony in relationships, which causes a decrease in the satisfaction with communication.

\section{Conclusion and future directions}

We believe that one of the most important aspects of this study was the demonstration of the utility of applying more general cultural theory to the study of organizational cultures. There is a positive cultural change process in Serbian organizations, creating a great challenge for learning processes. To attract, motivate, and retain talented managers, many organizations try to become employers of choice. Employers of choice achieve this reputation through innovative and compelling HR practices that benefit both employees and their organizations. Thus, it is critically important to monitor employees' job satisfaction level. Our results provide good empirical evidence that GLOBE organizational culture dimensions are significant predictors of all facets of job satisfaction and that relations between some GLOBE organizational culture dimensions, and facets of job satisfaction are moderated by the leader-member exchange (LMX) variable. We have proved that a high level of LMX may enhance the level of satisfaction with many aspects of the job of middle managers and that a good fit of "as it should be" GLOBE organizational culture dimensions and LMX is a very important factor for the job satisfaction of middle managers. Our results may help leaders to introduce changes of 
organizational culture through maximizing or minimizing certain cultural dimensions in order to increase the level of facets of job satisfaction. From a practical standpoint, the identification of an association between LMX practices and perceptions of organizational culture dimensions can help organizations diagnose their culture and identify potential levers for organizational change efforts.

The liberalization of the economy and the democratization of politics in the early 2000 s in Serbia entailed changes in the organizational culture. Nevertheless, several inherited features from the command economy organizational culture are still common for Serbian organizations (for example, a high power distance organizational culture). The dramatic increase in products, markets, enhanced technology, and robust competition has led to a dynamic global business environment. Serbian middle managers are aware of the need for change (a high "as it should be" future orientation), however, the challenge lies in implementing strategies that stick. The high level of expectation of employees in Serbian organizations for performance oriented organizational culture has to be followed by such performance oriented management approaches that align employees' incentive and accountability mechanisms with the goals of the organization. A key to redirecting employee performance toward organizational goals is to establish a performance oriented culture in which employees understand the importance of and the connection between their performance and the organization's success. The failure to involve staff constructively in an organization's efforts to become more performance based means running the risk that the changes will be more difficult and protracted than necessary.

\section{Limitations and Future Directions}

The fact that the data was collected in Serbia raises the question of the transferability of our results into other cultural regions. There is a high possibility of mergers and acquisitions of many organizations in the former Yugoslav republics in the near future in order to achieve a competitive advantage on the global market. Hence, it would be interesting to investigate the GLOBE organizational culture dimensions and the influence of the LMX relationship on the correlations between GLOBE organizational culture dimensions and facets of job satisfaction in organizations from these new countries.

We are thankful to both referees for their valuable suggestions which helped us to improve earlier versions of this paper.

This paper is a part of the research project no. 176020, which is funded by the Ministry of Science and Technological Development of the Republic of Serbia. 


\section{References}

Alas, R./Vadi, M. (2004): The impact of organisational culture on attitudes concerning postsoviet organizations. Journal for East European Management Studies. 9(1), 20-40.

Amos, E.A./Weathington, B.L. (2008): An analysis of the relation between employeeorganisation value congruence and employee attitudes. The Journal of Psychology. 142(6), 615-631.

Anseel, F./Lievens, F. (2007): The long-term impact of the feedback environment on job satisfaction: A field study in a Belgian context. Applied Psychology: An International Review. 56(2), 254-266.

Bakacsi, Gy. (1999). The Pendulum Effect: Culture, Transition, Learning in: Makó, Csaba Warhurst, Chris (eds.): The Management and Organisation of Firm in the Global Context. Published by the Institute of Management Education, University of Gödöllö and the Department of Management and Organisation, Budapest University of Economic Sciences, 111-118.

Bandura, A. (1977): Social Learning Theory. New York: General Learning Press.

Bellou, V. (2010): Organizational culture as a predictor of job satisfaction: the role of gender and age. Career Development International. 15(1), 4-19.

Bilsky. W./Jehn, K.A. (2002): Organizational culture and individual values: evidence for a common structure, in: Myrtek, M. (Ed.) Die Person im biologischen und sozialen Kontext, Göttingen: Hogrefe. 211-228.

Black, R.J. (2003): Organisational Culture: Creating the Influence Needed for Strategic Success. London UK.

Brown A. (1995): Organizational Culture. London: Pitman.

Calori, R./Sarnin, P. (1991): Corporate culture and economic performance: A French study. Organizational Studies. 12, 49-74.

Cameron, K.S./Quinn, R.E. (1999): Diagnosing and Changing Organizational Culture. OD Series: Addison-Wesley.

Chow, G.C. (1960): Tests of equality between sets of coefficients in two linear regression. Econometrica. 26(3), 591-605.

Collins, C.C./Porras, J.I. (1994): Built to Last: Successful Habits of Visionary Companies, Harper-Collins Publishers.

Conner, D. (1992): Managing at the speed of change. New York: Villard Books.

Crouch, A./Yetton, P. (1988): Manager-subordinate dyads: Relationships among task and social contact, manager friendliness, and subordinate performance in management groups. Organizational Behavior and Human Decision Processes. 41, 65-82.

Cummings, T.G./Worley, C.G. (2005): Organization development and change (8th ed.): Cincinnati, OH: South-Western Thomson.

Dansereau, F./Graen, G.B./Haga, W. (1975): A vertical dyad linkage approach to leadership in formal organizations. Organizational Behavior and Human Performance. 13, 46-78.

Denison, D. R. (1996): What is the difference between organizational culture and organizational climate? A native's point of view on a decade of paradigm wars. Academy of Management Review, 21: 619-654. 
Deshpande, R./Webster, F.E. Jr. (1989): Organization culture and marketing: Defining the research agenda. Journal of Marketing. 53(1), 3-15.

Dienesch, R.M./Liden, R.C. (1986): Leader-member exchange model of leadership: A critique and further development. Academy of Management Review. 11(3), 618-634.

Donnelly, R.M. (1984): The Interrelationship of Planning with, Corporate Culture in the Creation of Shared Values. Managerial Planning, May-June.

Erdogan, B./Liden, R.C./Kraimer, M.L. (2006): Justice and leader-member exchange: The moderating role of organizational culture. Academy of Management Journal. 49(2), 395-406.

Erdogan, B./Enders, J. (2007): Support from the top: supervisors' perceived organizational support as a moderator of leader-member exchange to satisfaction and performance relationships. Journal of Applied Psychology. 92(2), 321-330.

Eskildsen, J.K. /Kristensen, K./Westlund, A. (2004). Work motivation and job satisfaction in the Nordic countries. Employee Relations, Vol. 26, No. 2, 122-36.

Fairhurst, G.T. (1993): The leader-member exchange patterns of women leaders in industry: A discourse analysis. Communication Monograph. 60, 321-351.

Fairhurst, G.T./Chandler, T.A. (1989): Social structure in leader-member exchange interaction. Communication Monograph. 56, 215-239.

Fargher, S./Kesting, S./Lange, T./Pacheco, G. (2008): Cultural heritage and job satisfaction in Eastern and Western Europe. International Journal of Manpower. 29, 630-650.

Feichtinger, C./Fink, G. (1998): The collective culture shock in transition countries theoretical and empirical implications. Leadership \& Organization Development Journal. Vol. 19 Iss: 6,302 - 308 .

Gerstner, C.R./Day, D.V. (1997): Meta-analytic review of leader--member exchange theory: Correlates and construct issues. Journal of Applied Psychology. 82, 827-844.

Graen, G.B./Novak, M.A./Sommerkamp, P. (1982): The effects of leader-member exchange and job design on productivity and satisfaction: Testing a dual attachment model. Organizational Behavior and Human Performance. 30, 109-131.

Graen, G.B./Orris, D./Johnson, T. (1973): Role assimilation processes in a complex organization. Journal of Vocational Behavior. 3, 395-420.

Graen, G.B./Uhl-Bien, M. (1995): Relationships-Based Approach to Leadership: Development of Leader-Member Exchange (LMX) Theory of Leadership Over 25 Years: Applying a Multi-Level, Multi-Domain Approach. Leadership Quarterly. 6, 219-247.

Green, S.G./Anderson, S.E./Shivers, S.L. (1996): Demographic and organizational influences on leader-member exchange and related work attitudes. Organizational Behavior and Human Decision Processes. 66, 203-214.

Hofstede, G. (1980): Culture's Consequences - International Differences in Work-Related Values, Abridged Edition, Sage, Newbury Park.

Hofstede, G. (1991): Cultures and organizations: software of the mind. McGraw-Hill, London, New York. 
Hofstede, G. (1998): Attitudes, Values, and Organizational Culture: Disentangling the Concepts. Organization Studies. 19(3), 477-493.

Hofstede, G. (2001): Culture's Consequences, Comparing Values, Behaviors, Institutions, and Organizations Across Nations. Thousand Oaks CA: Sage Publications.

Hofstede, G./Hofstede, G.J. (2005): Cultures and Organizations: Software of the Mind, McGraw-Hill, New York, NY.

Hofstede, G./Neuijen, B./Ohavy, D.D./Sanders, G. (1990): Measuring organizational cultures: A qualitative and quantitative study across twenty cases, Administrative Science Quarterly. 35, 286-316.

House, R.J.,/Hanges, P.J./Javidan, M./Dorfman, P.W./Gupta. V. (2004): Leadership, culture, and organizations: The GLOBE study of 62 societies. Thousand Oaks, CA: Sage.

House, R.J./Hanges, P.J./Ruiz-Quintanilla, S.A./Dorfman, P.W./Javidan,M./Dickson, M.W./Gupta, et al. (1999): Cultural influences on leadership and organizations: Project GLOBE, in: Mobley, W.H. / Gessner, M.J./ Arnold, V. (Eds.), Advances in Global Leadership. (171-233). Stamford, CN: JAI

House, R.J./Javidan, M./Hanges, P./Dorfman, P. (2002): Understanding Cultures and Implicit Leadership Theories Across the Globe: An Introduction to Project GLOBE. Journal of World Business. 37(1), 3-10.

Huang, I.C. /Wu, J.M. (2000): The corporate culture and its effect on organizational commitment and job satisfaction in public sector: an example of the Taiwan Tobacco and Liquor Monopoly Bureau. Review of Public-owned Enterprises, Vol. 2 No. 1, $25-46$.

Hughes, L.W./Avey, J.B./Nixon, D.R. (2010): Relationships Between Leadership and Followers' Quitting Intentions and Job Search Behaviors. Journal of Leadership and Organizational Studies. 17(4), 351 - 362.

Javidan, M. /House, R.J. (2001): Cultural Acumen for the Global Manager: Lessons from Project GLOBE. Organizational Dynamics. Vol. 29 (4), p. 289-305.

Judge, T.A./Thoresen, C.J./Bono, J.E./Patton, G.K. (2001): The Job Satisfaction - Job Performance Relationship: a Qualitative and Quantitative Review. Psychological Bulletin. 127, 376-407.

Katrinli, A./Atabay, G./Gunay, G./Cangarl, B.G. (2010): Perception of organizational politics and LMX: Linkages in distributive justice and job satisfaction. African Journal of Business Management. 4(14), 3110-3121.

Kotter, J. P. (1996): Leading change. Boston: Harvard Business School Press.

Kotter, J./Heskett, J.L. (1992): Corporate Culture and Performance. The Free Press, New York.

Kroeber, A.L./Kluckhohn, C. (1952): Culture: A critical review of concepts and definitions. Harvard University Peabody Museum of American Archeology and Ethnology Papers 47.

Lauzen, M.M./Dozier, D.M. (1994): Issues management mediation of linkage between environmental complexity and management of the public relations function. Journal of Public Relations Research. 6(3), 163-184. 
Lee, Y.D./Chang, H.M. (2008): Relations between Team Work and Innovation in Organisations and the Job Satisfaction of Employees: A Factor Analytic Study. International Journal of Management. 25(3), 732-779.

Liden, R.C./Sparrowe, R.T./Wayne, S.J. (1997): Leader-member exchange theory. The past and potential for the future. Research in Personnel and Human Resources Management. 15, 47-119.

Liu, W. (2004): Perceived organizational support: linking human resource management practice with important work outcomes, PHD Thesis, the Faculty of the Graduate School of the University of Maryland, College Park.

http://drum.lib.umd.edu/bitstream/1903/1688/1/umi-umd-1658.pdf

Locke, E.A. (1976): The nature and causes of job satisfaction, in: M.D. Dunnette (Ed.), Handbook of industrial and organizational psychology. Chicago: Rand McNally.

Lund, D.B. (2003): Organizational culture and job satisfaction. Journal of Business \& Industrial Marketing. 18(3), 219-236.

McKinnon, L.J./Harrison, L.G./Chow, W.C. /Wu, A. (2003): Organizational culture: association with commitment, job satisfaction, propensity to remain and information sharing in Taiwan. International Journal of Business Studies. Vol. 11 No. 1, 25-44.

Meyer, J.P./Stanley, D.J./Herscovitch, L./Topolnytsky, L. (2002): Affective, Continuance, and Normative Commitment to the Organization: A Meta-analysis of Antecedents, Correlates, and Consequences. Journal of Vocational Behavior. 61, 20-52.

Mikl-Horke, G. (2004): Globalization, and the diffusion of management innovations. Journal for East European Management Studies. 9(2), 98-122.

Milin, P./Hadžić,O. (2011): Moderating and Mediating Variables in Psychological Research, in: Lovrić, M. (Ed.) International Encyclopedia of Statistical Science (849-852). Berlin: Springer.

Minkov, M. /Hofstede, G. (2011): The evolution of Hofstede's doctrine. Cross Cultural Management: An International Journal. Vol. 18, No. 1, 10-20.

Moynihan, D.P./Pandey, S.K. (2007): Finding workable levers over work motivation: Comparing job satisfaction, job involvement, and organizational commitment. Administration \& Society. 39(7), 803 - 832.

Ogbonna, E. (1993): Managing Organizational Culture: Fantasy or Reality?. Human Resource Management Journal. 2(3), 42-54.

Ogbonna, E./Harris, L.C. (2000): Leadership style, organizational culture and performance: empirical evidence from UK companies. The International Journal of Human Resource Management. 11, 766-788.

O'Reilly, C.A. III/Chatman, J./Caldwell, D.F. (1991): People and Organizational Culture: A Profile Comparison Approach to Assessing Person-Organization Fit. Academy of Management Journal. 34(3), 487-516.

Papalexandris, N./Panayotopoulou, L. (2004): Exploring the mutual interaction of societal culture and human resource management practices: Evidence from 19 countries. Employee Relations. 26(5), 495-509. 
Pellegrini, E.K./Scandura, T.A. (2006): Leader-Member Exchange (Lmx), Paternalism, and Delegation in the Turkish Business Culture: An Empirical Investigation. Journal of International Business Studies. 37(2), 264-279.

Pettigrew, A.M. (1979): On studying organizational culture. Administrative Science Quarterly. 24, 570-581.

Platonova, E.A./Hernandez, R.S./Shewchuk, R.M. /Leddy, K.M. (2006): Study of the relationship between organizational culture and organizational outcomes using hierarchical linear modeling methodology. Quality Management in Health Care. Vol. 15, No. 3, 200-209.

Scandura, T.A./Graen, G.B. (1984): Moderating effects of initial leader-member exchange status on the effects of a leadership intervention. Journal of Applied Psychology. 69, 428-436.

Schein, E.H. (1985): Organizational Culture and Leadership: A Dynamic View. San Francisco: Jossey-Bass Publishers.

Schein, E.H. (1999): The Corporate Culture Survival Guide. Jossey-Bass Publishers, San Francisco.

Sempane, M.E./Rieger, H.S./Roodt, G. (2002): Job Satisfaction In Relation To Organisational Culture. SA Journal of Industrial Psychology. 28(2), 23-30.

Settoon, R.P./Bennett, N./Liden, R.C. (1996): Social exchange in organizations: Perceived organizational support, leader-member exchange, and employee reciprocity. Journal of Applied Psychology. 81, 219-227.

Silverthorne, C. (2004): The impact of organizational culture and person-organization fit on organizational commitment and job satisfaction in Taiwan. Leadership \& Organization Development Journal , Vol. 25, 592-599.

Sousa-Poza, A. / Sousa-Poza, A.A. (2000). Wellbeing at work: a cross-national analysis of the levels and determinants of job satisfactio. Journal of Socio-Economics. Vol. 29 No. 6, 517-38.

Spector, P.E. (1985): Measurement of human service staff satisfaction: Development of the job satisfaction survey. American Journal of Community Psychology. 13(6), 693-713.

Spector, P.E. (1997): Job satisfaction: Application, assessment, cause, and consequences. Thousand Oaks, CA: Sage Publications, Inc.

Stepina, L.P./Perrewe, P.L./Hassell, B.L. (1991): A comparative test of the independent effects of interpersonal, task, and reward domains on personal and organizational outcomes. Journal of Social Behavior and Personality. 6, 93-104.

Stringer, L. (2006): The link between the quality of the supervisor-employee relationship and the level of the employee's job satisfaction. Public Organization Review. 6, 125-142.

Uhl-Bien, M. (2007): The Achilles' heel of LMX: Reexamining the measurement-theory link. Panelist, the Society for Industrial and Organizational Psychology meetings, New York.

Venaik S./Brewer, P.A. (2008): Contradictions in national culture: Hofstede vs GLOBE, in: Cantwell, J. /Kiyak, T.: Proceedings of the 50th Annual Meeting of the Academy of International Business. 50th Annual Meeting of the Academy of International Business (AIB), Milan, Italy. 274-274. 20 June - 3 July. 
Wallack, E.J. (1983): Individuals and Organizations: The Cultural Match. Training and Development Journal. 37, 29-36.

Wayne, S.J./Shore, L.M./Bommer, W.H./Tetrick, L.E. (2002): The role of fair treatment and rewards in perceptions of organizational support and leader-member exchange. Journal of Applied Psychology. 87, 590-598.

Xenikou, A./Furnham, A. (1996): A Correlational and Factor Analytic Study of Four Questionnaire Measures of Organizational Culture. Human Relations. Vol. 49, 349371.

Zhao, H. (2006): Expectations of recruiters and applicants in large cities of China. Journal of Managerial Psychology. 21(5), 459-475. 